\title{
Effect of Medications, Dietary Supplements and Psychosocial Interventions in Burning Mouth Syndrome Patients: A Systematic Review with Meta-analyses
}

\author{
Yen Phan $^{1}$, Richard Yeh ${ }^{1}$, Gil Colmenar ${ }^{1}$, Piedad Suarez-Durall ${ }^{2}$, Reyes Enciso ${ }^{2, *}$ \\ ${ }^{1}$ Herman Ostrow School of Dentistry, University of Southern California, USA \\ ${ }^{2}$ Division of Dental Public Health and Pediatric Dentistry, Herman Ostrow School of Dentistry, \\ University of Southern California, USA
}

Copyright $\bigcirc 2017$ by authors, all rights reserved. Authors agree that this article remains permanently open access under the terms of the Creative Commons Attribution License 4.0 International License

\begin{abstract}
The objective of this review is to determine the efficacy of cognitive behavioral therapy, oral and topical medications as well as dietary supplements to reduce pain in burning mouth syndrome patients (BMS). Methods: The Cochrane Library, Medline via PubMed and Web of Science were searched. Eligible studies were limited to randomized placebo-controlled trials on interventions used to treat BMS. Risk of bias was independently assessed in triplicate. Results: Twenty-two studies were included in this review. Fifteen reported baseline and post-treatment pain intensity, however due to the heterogeneity of the interventions subgroup analyses are presented by intervention. Subgroup analyses on the reduction of pain with alpha-lipoic acid (ALA) showed no significant difference with placebo $(p=.713)$. However, a subgroup analysis with four studies showed ALA improved patients' symptoms significantly ( $R R=2.676 ; 95 \%$ CI 1.933 to 3.705 ; $\mathrm{p}<.001)$. Individual studies showed significant improvement in pain intensity with topical clonazepam $(p<.001)$, capsaicin $(\mathrm{p}<.001)$, catuama $(\mathrm{p}=.009)$, and CBT $(\mathrm{p}<.001)$. No significant differences were found in individual studies reporting pain reduction with benzydamine $\mathrm{HCl}$, chamomile, H. perforatum, lidocaine, lycopene-enriched olive oil and trazodone $(\mathrm{p}>.05)$. Pooled results with two studies each showed capsaicin $(\mathrm{p}<.001)$ and CBT $(\mathrm{p}=.031)$ significantly improved patients' symptoms. Conclusions: Low quality of evidence is available due to heterogeneity of the interventions, unclear or high risk of bias and differences in outcomes reported resulting in small number of studies included in each subgroup analysis. ALA, topical clonazepam, CBT, catuama and capsaicin should be studied further in high quality randomized clinical trials to provide recommendations for BMS patients.
\end{abstract}

Keywords Burning Mouth Syndrome, Oral Medications, Clonazepam, Cognitive Behavioral Therapy, Alpha-Lipoic Acid

\section{Introduction}

There are an estimated 1.3 million Americans afflicted with Burning Mouth Syndrome (BMS) and many of these patients suffer from comorbidities such as anxiety, depression and psychological disorders [1]. Primary BMS, or essential/idiopathic BMS, is defined as a benign condition that presents as a burning sensation in the absence of any obvious findings in the mouth as well as abnormal blood tests [2]; in these patients, organic local/systemic causes cannot be identified [3]. Secondary BMS refers to the condition in which an identifiable pathology is causing the burning sensation [3]. Some of these pathologies include mucosal diseases (lichen planus, candidiasis, etc.), hormonal disturbances, diabetes, anemia, nutritional deficiencies and salivary gland dysfunction [4].

The symptom of burning sensation can involve the whole mouth or be localized to the lips or tongue [5]. Lauria et al. [6] concluded that "burning mouth syndrome is caused by a trigeminal small-fiber sensory neuropathy and that a superficial biopsy of the tongue can be helpful in assessing the diagnosis". BMS is a chronic pain disorder that could affect the patient's quality of life and $21 \%$ of patients may also develop psychological distress leading to enhanced pain perception [7]. It is often accompanied by subjective complaints of dysguesia and xerostomia with no identifiable dental or medical pathology [1].

Medications are often used to treat BMS, however, many have side-effects and potential psychological dependence therefore alternative treatments have been developed. The interventions included in this systematic review are cognitive behavioral therapy (CBT) [8, 9], systemic and topical medications as well as herbal supplements. (Table 1) 
Table 1. Interventions included in this systematic review.

\begin{tabular}{|c|c|}
\hline Intervention & Mechanism of action \\
\hline Cognitive behavioral therapy (CBT) $[8,9]$ & $\begin{array}{l}\text { CBT is a psychotherapy based upon behavioral and cognitive principles, used to treat anxiety and } \\
\text { depression and does not have side-effects. CBT can help the patient deal with the sense of } \\
\text { overwhelming problems by breaking them down in smaller parts. Biofeedback can help to monitor } \\
\text { physiological regulation and to learn coping strategies to change negative thoughts to positive } \\
\text { thoughts [34]. }\end{array}$ \\
\hline $\begin{array}{l}\text { Antioxidants - alpha lipoic acid (ALA) }[10- \\
16]\end{array}$ & $\begin{array}{l}\text { ALA is a potent antioxidant which decreases oxidative damage in the nervous system [10-15], has a } \\
\text { broad spectrum of action towards many free radical species and boosts the endogenous antioxidant } \\
\text { systems [30]. }\end{array}$ \\
\hline Anti-convulsants - gabapentin [13] & $\begin{array}{l}\text { Gabapentin is an anticonvulsant which acts as an agonist of the inhibitory neurotransmitter GABA of } \\
\text { central activity [13]. }\end{array}$ \\
\hline Benzodiazepines - clonazepam [17-19] & Topical clonazepam may have an effect on GABA receptors in the peripheral tissues [19]. \\
\hline \multicolumn{2}{|l|}{ Analgesics } \\
\hline Bupivacaine [20] & $\begin{array}{l}\text { Bupivacaine is also a local anesthetic agent that can be used in patients with BMS based on the } \\
\text { hypothesis that it can alleviate the symptoms of oral burning pain, xerostomia and taste alterations in } \\
\text { these patients [20]. }\end{array}$ \\
\hline Lidocaine [21] & $\begin{array}{l}\text { Lidocaine is a common local anesthetic agent that blocks signal conduction in neurons (sodium } \\
\text { channel blocker); topical lidocaine is useful in the management of peripheral neuropathic pain having } \\
\text { the advantage that systemic side effects are extremely rare because of its minimal blood levels } \\
\text { presence [31,32]. }\end{array}$ \\
\hline NSAIDs - benzydamine hydrochloride [22] & $\begin{array}{l}\text { Benzydamine hydro-chloride }(\mathrm{Bzd} \mathrm{HCl}) \text { is a nonsteroidal drug with analgesic and anti-inflammatory } \\
\text { properties [22]. }\end{array}$ \\
\hline Antidepressants - trazodone [23] & $\begin{array}{l}\text { Trazodone is a triazolopyridine derivative that is chemically and pharmacologically unrelated to other } \\
\text { currently available antidepressants [23]. }\end{array}$ \\
\hline \multicolumn{2}{|l|}{ Dietary supplements } \\
\hline St John's Wort [24] & $\begin{array}{l}\text { Hypericum perforatum (St John's Wort) has been used in the treatment of mild to moderate forms of } \\
\text { depression in many countries [24]. }\end{array}$ \\
\hline Capsaicin $[16,25,26]$ & $\begin{array}{l}\text { Capsaicin is the burning component in hot chili pepper, binds to the TRPV-1 calcium } \\
\text { channel-receptor [33], desensitizes type } \mathrm{C} \text { receptors for pain and reduces neurogenic inflammation } \\
\text { [25]. One clinical study showed that systemic administration of capsaicin was effective to treat BMS } \\
\text { however it is also associated with significant gastric pain [25]. }\end{array}$ \\
\hline Catuama [27] & $\begin{array}{l}\text { Catuama, an herbal product from Brazil, contains four extracts of medicinal plants with analgesic, } \\
\text { antibacterial, and vast-relaxant effects among others [27]. }\end{array}$ \\
\hline Topical chamomile [28] & $\begin{array}{l}\text { Chamomile has a variety of active flavonoids, especially apigenin, which is a selective COX-2 } \\
\text { inhibitor and possesses anti-inflammatory and pain relief effects therefore it has beneficial } \\
\text { therapeutic effects on BMS symptoms, with a few adverse effects [28]. }\end{array}$ \\
\hline Lycopene-enriched virgin olive oil [29] & $\begin{array}{l}\text { Lycopene-enriched virgin olive oil is a carotenoid synthesized by plants and microorganisms that is } \\
\text { found in tomatoes and acts as an antioxidant with the potential to play a protective role in chronic } \\
\text { human diseases including cancer [29]. }\end{array}$ \\
\hline
\end{tabular}

There is only limited evidence to guide the clinician in the management of BMS [7]. This systematic review was designed to analyze the randomized controlled trials (RCTs) regarding the efficacy of oral and topical medications and herbal supplements, as well as CBT, in the management of primary BMS.

\section{Materials and Methods}

\subsection{Criteria for Considering Studies for this Review}

Studies were limited to randomized placebo-controlled trials on the efficacy of oral systemic, topical medications or psychosocial interventions compared to placebo conducted in patients diagnosed with BMS. Studies included at least one clinical endpoint reported by trial authors. Editorials/commentaries, reviews, case studies, animal studies, cost-effectiveness studies, and clinical guidelines were rejected. Reviews were scanned for relevant trials. Three authors (Y.P., R.Y. and G.C.) individually assessed trial reports to determine eligibility. Disagreements were resolved by discussion with a fourth reviewer (R.E.) taking the final decision in case of lack of agreement after discussion. Articles not available in English and studies conducted in children $(\leq 18$ years of age) were not included. Primary outcomes were pain intensity (short and/or long term) measured using VAS (Visual Analog Scale) or a Numeric Rating Scale (NRS), also named Visual Numeric Scale (VNS). The VAS is usually a $0-10 \mathrm{~cm}$ or $0-100 \mathrm{~mm}$ long straight line, marked at each end with labels which anchor the scale for instance, $0=$ "no pain" and 100 = "worst imaginable pain". Respondents are asked to place a line perpendicular to the VAS line at the point that represents their pain intensity at that moment [35]. The NRS or VNS is a 11,21 or 101 points scale in which a respondent selects a whole number $(0-10$ or $0-20$ or $0-100$ integers, respectively) instead of placing a line. The end 
points of the NRS represent the extremes of no pain and worst imaginable pain [36]. Secondary outcomes were improvement of patient's symptoms and the number of responders (patients with at least $50 \%$ improvement in pain intensity measured with a visual or numeric scale).

\subsection{Search Strategies}

For the identification of eligible studies the following electronic databases were searched:

MEDLINE via PubMed (searched on 1/27/2016 and updated on 2/2/2017) using the following search strategy: ("Burning Mouth Syndrome/therapy"[Mesh] OR "burning mouth syndrome" OR "oral dysesthesia" OR glossodynia OR stomatodynia OR glossopyrosis) AND (drug therap* OR topical medication* OR systemic medication* OR oral medication OR medication* OR gabapentin OR pregabalin OR clonazepam OR lidocaine OR doxepin OR capsaicin OR "alpha-lipoic acid" OR ALA OR Amisulpride OR paroxetine OR clordemetildiazepam OR amitriptyline OR Duloxetine OR behavior* therapy* OR psychotherapy* OR psychoanal* OR psychological therapy* OR hypnosis OR cognitive behavior* therapy*). Filters: Humans, English

The Web of Science (searched on 1/27/2016 and updated on $2 / 2 / 2017$ using the following search strategy:

TOPIC ("burning mouth syndrome" OR "oral dysesthesia" OR glossodynia OR stomatodynia OR glossopyrosis) $A N D$ TOPIC: (drug therap* OR topical medication* OR systemic medication* OR oral medication OR medication* OR gabapentin OR pregabalin OR clonazepam OR lidocaine OR doxepin OR capsaicin OR "alpha-lipoic acid" OR ALA OR Amisulpride OR paroxetine OR clordemetildiazepam OR amitriptyline OR Duloxetine OR behavior* therapy* OR psychotherapy* OR psychoanal* OR psychological therapy* OR hypnosis OR cognitive behavior* therapy*)

The Cochrane Library (searched on 1/27/2016 and updated on 2/2/2017) using the following search strategy:

("burning mouth syndrome" or "oral dysesthesia" or glossodynia or stomatodynia or glossopyrosis) AND (drug therap* or topical medication* or systemic medication* or oral medication or medication* or gabapentin or pregabalin or clonazepam or lidocaine or doxepin or capsaicin or "alpha-lipoic acid" or ALA or Amisulpride or paroxetine or clordemetildiazepam or amitriptyline or Duloxetine or behavior* therapy* or psychotherapy* or psychoanal* or psychological therapy* or hypnosis or cognitive behavior* therapy*)

The reference lists of all eligible RCT's, reviews and systematic reviews were checked by three review authors for additional studies. The Preferred Reporting Items for Systematic Reviews and Meta-Analyses (PRISMA) [37] flow chart was used.

\subsection{Selection of Studies and Data Extraction}

The title and abstracts of the articles resulting from the search strategy were screened independently by three review authors (G.C., R.Y., Y.P.) for their inclusion. Disagreements were resolved by a fourth author (R.E.). If the abstracts of the articles met the inclusion criteria or a clear decision could be made from the title or abstract, full articles were obtained and reviewed by two authors. Studies rejected were recorded along with reasons for their exclusion. The reviewers extracted data from the eligible studies including the characteristics of trial participants, interventions, control groups if appropriate and outcomes.

\subsection{Assessment of Risk of Bias in Included Studies}

The assessment of risk of bias in the included RCTs was based on the approach described in the Cochrane Handbook for Systematic Reviews of Interventions [38]. A risk of bias table was completed for each included study.

\subsection{Measures of Treatment Effect}

For continuous outcomes of intensity of pain at baseline and post-treatment using different scales, we used the standardized differences in means (SDM) with 95\% confidence intervals to report pooled results. Risk ratios (RR) with $95 \%$ Confidence Intervals (CI) were reported for two dichotomous variables: patient's improvement of overall symptoms and $50 \%$ reduction in pain intensity. The analyses included only the available data (ignoring missing data). We calculated standard deviations for baseline and post-treatment pain intensity for one study based on length of error bars in graphs and a ruler. No other statistical methods to account for missing data were used.

The criteria for pooling studies was based on similar characteristics of the patients (primary BMS), intervention and outcome measures. Statistical heterogeneity was assessed using the Cochran's test for heterogeneity [39] and quantified by the $\mathrm{I}^{2}$ statistic [40]. Meta-analyses were carried out for studies reporting similar outcome measures. When appropriate, estimates of effect were combined using a random-effects model if statistical heterogeneity was found, otherwise the fixed-effect model was used. Subgroup analyses were conducted in this review for each type of intervention. Statistical analyses were performed using Comprehensive Meta-analysis v3 software (Biostat, Englewood, NJ, USA).

\section{Results}

The searches resulted in 318 unduplicated references which were reduced to 23 relevant manuscripts based on the abstracts and titles. Main reasons for exclusion were: patients were not diagnosed with BMS $(\mathrm{n}=95)$, articles were not written in English $(\mathrm{n}=3)$, reviews $(\mathrm{n}=89)$, not a RCT $(\mathrm{n}=18)$, animal studies $(\mathrm{n}=2)$, abstract $(\mathrm{n}=7)$, editorial letter $(n=5)$, different therapeutic intervention $(n=14)$, open study $(n=12)$, book chapter $(n=4)$, case study $(n=11)$ and 
no control group $(\mathrm{n}=6)$, different outcome $(\mathrm{n}=19)$, protocol $(\mathrm{n}=4)$, pilot study $(\mathrm{n}=4)$ and case control $(\mathrm{n}=2)$. All 23 manuscripts identified were searched for full-text and analyzed for inclusion and one manuscript was eliminated (not BMS patients), which resulted in 22 manuscripts relevant for inclusion. The PRISMA flowchart shows a summary of our search results (Figure 1).

\subsection{Included studies}

Of the 22 included trials, two trials investigated the efficacy of CBT for treatment of burning mouth syndrome [8, 9]. Other studies researched the efficacy of oral and topical medications: seven trials of ALA [10-16], three clonazepam trials [17-19], three capsaicin studies (two peripheral mouth rinse and one oral systemic) $[16,25,26]$, one NSAID benzydamine [22], one antidepressant - trazadone [23], two local anesthetics (lingual nerve block with lidocaine [21]; bupivacaine lozenge [20]), and one anticonvulsant - gabapentin [13]. Three trials included herbal supplements: hypericum perforatum extract [24], catuama [27], topical chamomile [28] and lastly, lycopene-enriched virgin olive oil [29]. Five studies followed a crossover trial design [11, 20, $21,26,28]$ and all seventeen trials were randomized placebo-controlled trials (Table 2).

All 22 studies included control groups receiving a placebo therapy for comparison. One study on the efficacy of cognitive therapy used a second group receiving attention placebo therapy [8] while the other study used placebo pills in the control group [9]. Eighteen studies used placebo pills (i.e. starch and cellulose) which looked as the treatment therapy; one topical study used saline injection for comparison against lidocaine [21]; one study used water and dye identical to study product (olive oil) [29]. Valenzuela et al. [28] used a placebo that consisted of the same excipients and composition except that the experimental gel included chamomile $2 \%$ and the placebo did not.
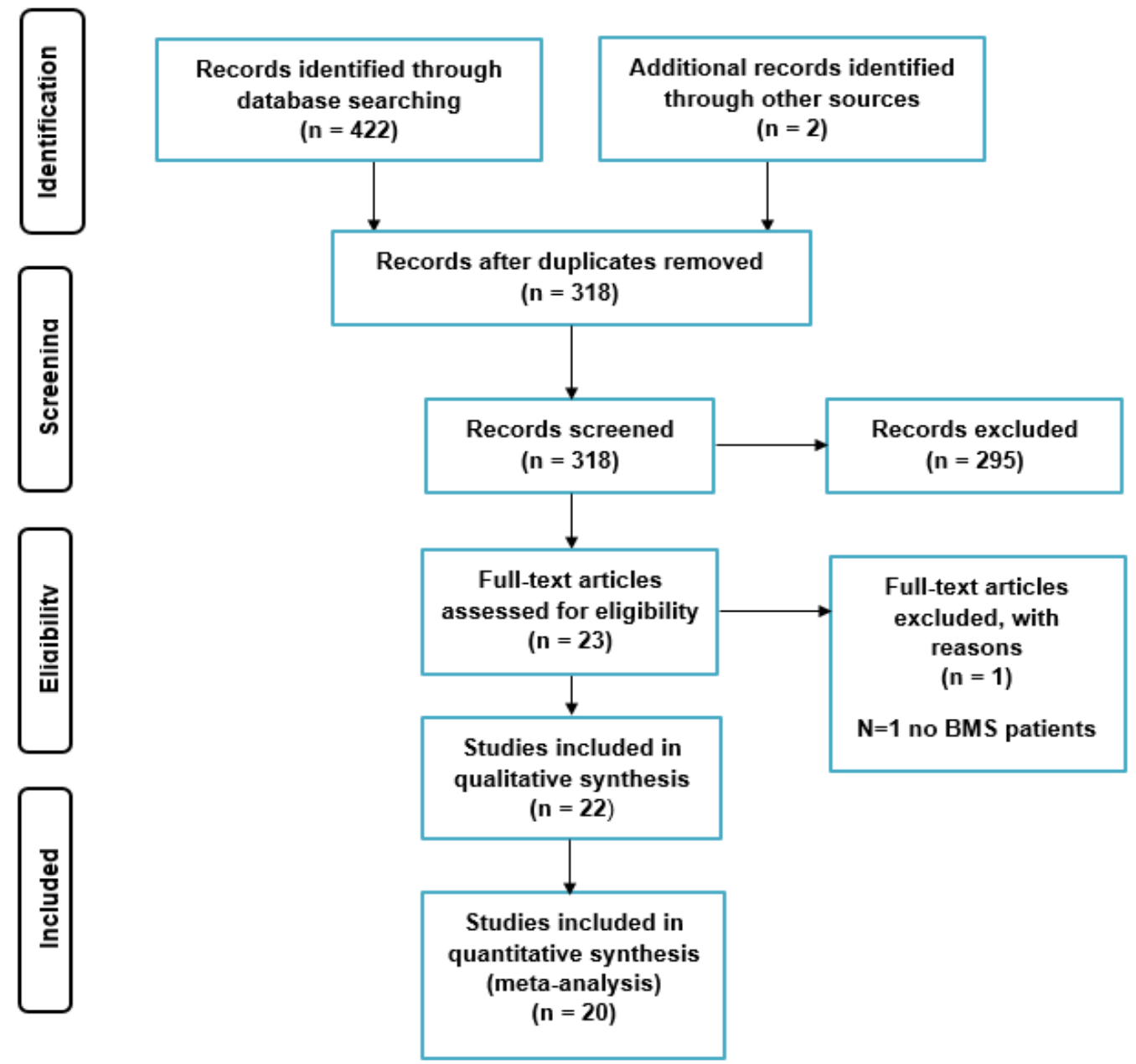

Figure 1. Preferred Reporting Items for Systematic Reviews and Meta-Analyses (PRISMA) flow chart 
Table 2. Summary of Eligible Studies

\begin{tabular}{|c|c|c|c|c|}
\hline Reference & $\begin{array}{l}\text { Year, Country } \\
\text { Sample Size }\end{array}$ & Intervention Groups & Gender and age & Study Type \\
\hline Bergdahl et al [8] & $\begin{array}{l}\text { 1995, Denmark, } \\
\qquad \mathrm{N}=30\end{array}$ & $\begin{array}{l}\text { Cognitive therapy group } \\
\qquad(\mathrm{n}=15) \\
\text { Attention Placebo group } \\
\quad(\mathrm{n}=15)\end{array}$ & $\begin{array}{c}24 \mathrm{~F} / 6 \mathrm{M} \\
\text { Female: mean age of } 56 \text { years } \\
\text { (range: } 40-69 \text { years) } \\
\text { Male: mean age of } 46 \mathrm{yr} \text { (range: } \\
\text { 38-57 yr). }\end{array}$ & $\mathrm{RCT}$ \\
\hline Cano-Carrillo et al [29] & $\begin{array}{l}\text { 2014, Spain } \\
\quad \mathrm{N}=60\end{array}$ & $\begin{array}{l}\text { Lycopene-enriched olive } \\
\text { oil }(\mathrm{n}=30) \\
\text { Placebo(n=30) }\end{array}$ & $\begin{array}{c}\text { Olive oil: } 21 \mathrm{~F} / 9 \mathrm{M} \\
61.7 \pm 11.6 \text { years } \\
\text { Placebo: } 27 / 3 \mathrm{M} \\
64.9 \pm 14.1 \\
\end{array}$ & $\mathrm{RCT}$ \\
\hline Carbone et al [10] & $\begin{array}{l}2009, \text { Italy } \\
\quad \mathrm{N}=66\end{array}$ & $\begin{array}{c}\text { ALA }(\mathrm{n}=22) \\
\text { ALA }+ \text { Vitamins }(\mathrm{n}=22) \\
\text { Placebo }(\mathrm{n}=22)\end{array}$ & $\begin{array}{c}54 \mathrm{~F} / 12 \mathrm{M} \\
67.3 \pm 11.9 \text { years }\end{array}$ & $\mathrm{RCT}$ \\
\hline $\begin{array}{l}\text { Cavalcanti \& da } \\
\text { Silveira [11] }\end{array}$ & $\begin{array}{l}\text { 2009, Brazil, } \\
\quad \mathrm{N}=38\end{array}$ & $\begin{array}{c}\text { ALA }(n=19) \\
\text { Placebo }(n=19)\end{array}$ & $\begin{array}{c}34 \mathrm{~F} / 4 \mathrm{M} \\
\text { median } 62.9 \text { years } \\
\text { (range } 36-78 \text { years) }\end{array}$ & Crossover \\
\hline Femiano \& Scully [12] & $\begin{array}{l}\text { 2002, Location not } \\
\text { mentioned, } N=60\end{array}$ & $\begin{array}{c}\text { ALA }(n=30) \\
\text { Placebo }(n=30)\end{array}$ & $\begin{array}{c}42 \mathrm{~F} / 18 \mathrm{M} \\
\text { median 45years } \\
\text { (range 22-68years) }\end{array}$ & $\mathrm{RCT}$ \\
\hline $\begin{array}{l}\text { Gremeau-Richard et al } \\
{[19]}\end{array}$ & $\begin{array}{l}\text { 2004, France, } \\
\qquad \mathrm{N}=48\end{array}$ & $\begin{array}{l}\text { Tyopical Clonazepam } \\
(\mathrm{n}=24) \\
\text { Placebo }(\mathrm{n}=24)\end{array}$ & $\begin{array}{c}44 \mathrm{~F} / 4 \mathrm{M} \\
65 \pm 2.1 \text { years }\end{array}$ & $\mathrm{RCT}$ \\
\hline $\begin{array}{c}\text { Gremeau-Richard et al } \\
{[21]}\end{array}$ & $\begin{array}{l}\text { 2010, France, } \\
\mathrm{N}=20\end{array}$ & $\begin{array}{c}\text { Lidocaine }(n=20) \\
\text { Placebo saline }(n=20)\end{array}$ & $\begin{array}{c}20 \mathrm{~F} / 0 \mathrm{M} \\
\text { Over } 50 \text { years old }\end{array}$ & Crossover \\
\hline Heckmann et al [17] & $\begin{array}{l}\text { 2012, Germany, } \\
\mathrm{N}=20\end{array}$ & $\begin{array}{l}\text { Systemic Clonazepam } \\
(\mathrm{n}=10) \\
\text { Placebo }(\mathrm{n}=10)\end{array}$ & $\begin{array}{c}\text { Clonazepam: } 5 \mathrm{~F} / 5 \mathrm{M} \\
\text { Placebo: } 8 \mathrm{~F} / 2 \mathrm{M} \\
\text { mean age, } 67.5 \text { years (range } 49-89 \\
\text { years) }\end{array}$ & $\mathrm{RCT}$ \\
\hline $\begin{array}{l}\text { Lopez-D'Alessandro \& } \\
\text { Escovich [13] }\end{array}$ & $\begin{array}{l}\text { 2011, Argentina, } \\
\qquad \mathrm{N}=120\end{array}$ & $\begin{array}{c}600 \mathrm{mg} / \text { day of ALA } \\
(\mathrm{n}=20) \\
300 \mathrm{mg} / \text { day gabapentin } \\
(\mathrm{n}=20) \\
\text { combination of both drugs } \\
(\mathrm{n}=20) \\
\text { Placebo }(\mathrm{n}=60)\end{array}$ & $\begin{array}{c}94 \mathrm{~F} / 26 \mathrm{M} \\
14.1 \pm 57.5 \text { years }\end{array}$ & $\mathrm{RCT}$ \\
\hline Lopez-Jornet et al.,[14] & $\begin{array}{l}\text { 2009, Spain, } \\
\quad \mathrm{N}=60\end{array}$ & $\begin{array}{l}\text { Alpha Lipoic Acid }(\mathrm{n}=30) \\
\text { Placebo }(\mathrm{n}=30)\end{array}$ & $\begin{array}{c}54 \mathrm{~F} / 6 \mathrm{M} \\
64.37 \pm 11.61 \text { years }\end{array}$ & $\mathrm{RCT}$ \\
\hline Marino et al..,[16] & $\begin{array}{l}\text { 2010, Italy, } \\
\quad \mathrm{N}=56\end{array}$ & $\begin{array}{l}\text { Topical rinse of capsaicin } \\
\qquad(\mathrm{n}=14) \\
\text { ALA }(\mathrm{n}=14) \\
\text { Lyso }(\mathrm{n}=14) \\
\text { Placebo }(\mathrm{n}=14)\end{array}$ & $\begin{array}{c}56 \mathrm{~F} / 10 \mathrm{M} \\
62 \pm 9.8 \text { years }\end{array}$ & $\mathrm{RCT}$ \\
\hline Miziara et al.,[9] & $\begin{array}{l}\text { 2009, Brazil } \\
\quad \mathrm{N}=44\end{array}$ & $\begin{array}{l}\text { Group Psychotherapy } \\
\text { group }(n=24) \\
\text { Placebo pill group }(n=20)\end{array}$ & $\begin{array}{c}29 \mathrm{~F} / 15 \mathrm{M} \\
55 \pm 6.7 \text { years }\end{array}$ & $\mathrm{RCT}$ \\
\hline $\begin{array}{l}\text { Palacios-Sanchez et } \\
\text { al.,[15] }\end{array}$ & $\begin{array}{l}\text { 2015, Spain } \\
\quad \mathrm{N}=60\end{array}$ & $\begin{array}{l}\text { Systemic ALA }(\mathrm{n}=30) \\
\text { Placebo }(\mathrm{n}=30)\end{array}$ & $\begin{array}{c}55 \mathrm{~F} / 5 \mathrm{M} \\
\text { median age of } 62.13 \text { years (range } \\
36-86 \text { ) }\end{array}$ & $\mathrm{RCT}$ \\
\hline Petruzzi et al., [25] & $\begin{array}{l}\text { 2004, Italy, } \\
\quad \mathrm{N}=50\end{array}$ & $\begin{array}{l}\text { Oral capsaicin }(n=25) \\
\quad \text { Placebo }(n=25)\end{array}$ & $\begin{array}{c}\text { Capsaicin: } 17 \mathrm{~F} / 8 \mathrm{M} \\
55.6 \pm 6 \\
\text { Placebo: } 19 \mathrm{~F} / 6 \mathrm{M} \\
57.4 \pm 7\end{array}$ & $\mathrm{RCT}$ \\
\hline $\begin{array}{c}\text { Rodriguez de } \\
\text { Rivera-Campillo et } \\
\text { al.,[18] }\end{array}$ & $\begin{array}{l}\text { 2010, Spain, } \\
\quad \mathrm{N}=66\end{array}$ & $\begin{array}{c}\text { Topical Clonazepam } \\
(\mathrm{n}=33) \\
\text { Placebo }(\mathrm{n}=33)\end{array}$ & $\begin{array}{c}64 \mathrm{~F} / 2 \mathrm{M} \\
\text { Mean } 64.9 \text { years } \\
\text { (range } 48-85 \text { ) } \\
\end{array}$ & $\mathrm{RCT}$ \\
\hline Sardella et al., [22] & $\begin{array}{l}\text { 1999, Italy, } \\
\quad \mathrm{N}=30\end{array}$ & $\begin{array}{c}\text { Benzydamine } \mathrm{HCl}(\mathrm{n}=10) \\
\text { Placebo }(\mathrm{n}=10) \\
\text { no therapy }(\mathrm{n}=10) \\
\end{array}$ & $\begin{array}{l}26 \mathrm{~F} / 4 \mathrm{M} \\
\text { Mean } 69 \text { years } \\
\text { (range } 54-85 \text { ) } \\
\end{array}$ & $\mathrm{RCT}$ \\
\hline Sardella et al., [24] & $\begin{array}{l}\text { 2008, Italy, } \\
\quad \mathrm{N}=43\end{array}$ & $\begin{array}{l}\text { 300mg capsules of } \mathrm{H} . \\
\text { perforatum extract }(\mathrm{n}=21) \\
\text { Placebo }(\mathrm{n}=22)\end{array}$ & $\begin{array}{c}\text { H. perf: } 18 \mathrm{~F} / 1 \mathrm{M} \\
65.9 \pm 4.2 \\
\text { Placebo: } 17 \mathrm{~F} / 3 \mathrm{M} \\
63.9 \pm 4.9\end{array}$ & $\mathrm{RCT}$ \\
\hline Silvestre et al.,[26] & $\begin{array}{l}\text { 2012, Spain, } \\
\quad \mathrm{N}=23\end{array}$ & $\begin{array}{l}\text { Capsaicin rinse }(\mathrm{n}=12) \\
\text { placebo }(\mathrm{n}=11)\end{array}$ & $\begin{array}{c}19 \mathrm{~F} / 4 \mathrm{M} \\
72.65 \pm 12.10 \text { years } \\
(\text { range } 40-90)\end{array}$ & Crossover \\
\hline
\end{tabular}


Effect of Medications, Dietary Supplements and Psychosocial Interventions in Burning Mouth Syndrome Patients.

A Systematic Review with Meta-analyses.

\begin{tabular}{|c|c|c|c|c|}
\hline Spanemberg et al., [27] & $\begin{array}{l}\text { 2012, Brazil, } \\
\qquad \mathrm{N}=60\end{array}$ & $\begin{array}{l}\text { Catuama capsule of } 310 \mathrm{mg} \\
\qquad \begin{array}{c}(\mathrm{n}=30) \\
\text { Placebo }(\mathrm{n}=30)\end{array}\end{array}$ & $\begin{array}{c}\text { Catuama: } 27 \mathrm{~F} / 3 \mathrm{M} \\
63.6 \pm 9.61 \\
\text { Placebo: } 26 \mathrm{~F} / 4 \mathrm{M} \\
61.5 \pm 6.76\end{array}$ & $\mathrm{RCT}$ \\
\hline $\begin{array}{l}\text { Tammiala-Salonen \& } \\
\text { Forssell,[23] }\end{array}$ & $\begin{array}{l}\text { 1999, Finland, } \\
\mathrm{N}=37\end{array}$ & $\begin{array}{c}\text { Trazodone }(\mathrm{n}=18) \\
\text { Placebo }(\mathrm{n}=19)\end{array}$ & $\begin{array}{c}37 \mathrm{~F} / 0 \mathrm{M} \\
\text { Trazodone: mean } 61.1 \text { years } \\
\text { Placebo: } 56.2 \text { years } \\
\end{array}$ & $\mathrm{RCT}$ \\
\hline Treldal et al.,[20] & $\begin{array}{l}\text { 2016, Denmark } \\
\qquad \mathrm{N}=18\end{array}$ & $\begin{array}{l}\text { Topical Bupivacaine } \\
\text { (anesthetic) } \\
\mathrm{N}=9 \\
\text { Placebo, }(\mathrm{n}=9)\end{array}$ & $\begin{array}{c}14 \mathrm{~F} / 4 \mathrm{M} \\
\text { Median } 60 \text { years } \\
\text { (range } 39-71)\end{array}$ & Crossover \\
\hline Valenzuela et al., [28] & $\begin{array}{l}\text { 2015, Spain } \\
\quad \mathrm{N}=62\end{array}$ & $\begin{array}{l}\text { Topical Chamomile }(\mathrm{n}=31) \\
\text { Placebo }(\mathrm{n}=26)\end{array}$ & $\begin{array}{c}\text { Chamomile: } 28 \mathrm{~F} / 3 \mathrm{M} \\
65.8 \pm 10.6 \\
\text { Placebo: } 22 \mathrm{~F} / 4 \mathrm{M} \\
67.2 \pm 12.6 \\
\end{array}$ & Crossover \\
\hline
\end{tabular}

Table 3. Primary outcome: Change in pain intensity from baseline.

\begin{tabular}{|c|c|c|c|c|c|c|c|}
\hline & & \multicolumn{3}{|c|}{ TREATMENT GROUP } & \multicolumn{3}{|c|}{ PLACEBO GROUP } \\
\hline $\begin{array}{c}\text { Study } \\
\text { (Intervention/route) }\end{array}$ & $\begin{array}{c}\text { Scale } \\
\text { (Tx length) }\end{array}$ & $\begin{array}{c}\text { Pre-Tx } \\
\text { Mean } \pm \text { SD }\end{array}$ & $\begin{array}{c}\text { Post-Tx } \\
\text { Mean } \pm \text { SD }\end{array}$ & Sample size & $\begin{array}{c}\text { Pre-Tx } \\
\text { Mean } \pm \text { SD }\end{array}$ & $\begin{array}{c}\text { Post-Tx } \\
\text { Mean } \pm \text { SD }\end{array}$ & $\begin{array}{c}\text { Sample } \\
\text { size }\end{array}$ \\
\hline $\begin{array}{l}\text { Bergdahl et al.,[8] } \\
\text { (1995) (CBT) }\end{array}$ & $\begin{array}{c}\text { 0-7 VAS } \\
(12-15 \\
\text { weeks) }\end{array}$ & $5.0 \pm 0.8$ & $2.2 \pm 1$ & 15 & $4.3 \pm 1.7$ & $4.6 \pm 1.7$ & 15 \\
\hline $\begin{array}{l}\text { Cano-Carrillo et al.,[29] } \\
\text { (2014) (olive oil) }\end{array}$ & $\begin{array}{l}0-10 \text { VAS } \\
(12 \text { weeks })\end{array}$ & $8.25 \pm 1.25 ¥$ & $6.25 \pm 1.75 ¥$ & 26 & $8.5 \pm 1.0 ¥$ & $6 \pm 2 ¥$ & 24 \\
\hline $\begin{array}{c}\text { Carbone et al., [10] } \\
(2009)(\text { ALA) }\end{array}$ & $\begin{array}{l}\text { 0-10 VAS } \\
(2 \mathrm{mo} .)\end{array}$ & $6.50 \pm 2.59$ & $4.71 \pm 3.10$ & 14 & $6.65 \pm 2.41$ & $5.05 \pm 3.39$ & 20 \\
\hline $\begin{array}{c}\text { Gremeau-Richard et al., } \\
{[19](2004)} \\
\text { (clonazepam/topical) }\end{array}$ & $\begin{array}{l}0-10 \mathrm{NRS} \\
(2 \text { weeks })\end{array}$ & $6.0 \pm 0.3$ & $3.8 \pm 0.6$ & 24 & $6.0 \pm 0.3$ & $5.4 \pm 0.4$ & 24 \\
\hline $\begin{array}{c}\text { Gremeau-Richard et } \\
\text { al.,[21] (2010) } \\
\text { (lidocaine) }\end{array}$ & $\begin{array}{l}0-10 \text { VAS } \\
(2 \text { weeks })\end{array}$ & $5.6 \pm 2.8$ & $2.9 \pm 2.6$ & 20 & $5.0 \pm 2.4$ & $3.0 \pm 2.8$ & 20 \\
\hline $\begin{array}{c}\text { Heckmann et al.,[17] } \\
\text { (2012) } \\
\text { (clonazepam/oral) }\end{array}$ & $\begin{array}{l}0-10 \mathrm{NRS} \\
(6 \text { weeks) }\end{array}$ & $4.1 \pm 3.0$ & $3.9 \pm 2.9$ & 10 & $5.0 \pm 2.0$ & $4.6 \pm 2.4$ & 10 \\
\hline $\begin{array}{l}\text { Lopez-Jornet et al.,[14] } \\
\text { (2009) (ALA) }\end{array}$ & $\begin{array}{l}0-10 \text { VAS } \\
(2 \mathrm{mo} .)\end{array}$ & $\begin{array}{c}1 \text { month } \\
6.3 \pm 2.8 \\
2 \text { months } \\
6.3 \pm 2.8 \\
\end{array}$ & $\begin{array}{c}1 \text { month } \\
4.5 \pm 3.0 \\
2 \text { months } \\
4.0 \pm 2.7 \\
\end{array}$ & 23 & $\begin{array}{c}1 \text { month } \\
6.6 \pm 2.5 \\
2 \text { months } \\
6.6 \pm 2.5 \\
\end{array}$ & $\begin{array}{c}1 \text { month } \\
4.0 \pm 2.5 \\
2 \text { months } \\
2.8 \pm 2.5 \\
\end{array}$ & 16 \\
\hline $\begin{array}{l}\text { Marino et al.,[16] } \\
\text { (2010) (capsaicin) }\end{array}$ & $\begin{array}{l}0-10 \text { VAS } \\
\text { (8 weeks) }\end{array}$ & $6.1 \pm 2.2$ & $2.9 \pm 2.6$ & 14 & $4.8 \pm 2.3$ & $5.3 \pm 2.2$ & 14 \\
\hline $\begin{array}{l}\text { Marino et al.,[16] } \\
\text { (2010) (ALA) }\end{array}$ & $\begin{array}{l}0 \text {-10 VAS } \\
\text { (8 weeks) }\end{array}$ & $5.8 \pm 2.2$ & $3.7 \pm 2.5$ & 14 & $4.8 \pm 2.3$ & $5.3 \pm 2.2$ & 14 \\
\hline $\begin{array}{c}\text { Rodriguez de } \\
\text { Rivera-Campillo et } \\
\text { al.,[18] (2010) (Topical } \\
\text { Clonazepam) }\end{array}$ & $\begin{array}{l}0-10 \text { VAS } \\
\text { (1month) }\end{array}$ & $7.70 \pm 1.53$ & $2.85 \pm 1.70$ & 33 & $7.58 \pm 1.58$ & $4.24 \pm 1.20$ & 33 \\
\hline $\begin{array}{c}\text { Sardella et al. [22] } \\
(1999) \\
\text { benzydamine } \mathrm{HCl}\end{array}$ & $0-10$ VAS & $6.7 \pm 1.16$ & $6.1 \pm 1.20$ & 10 & $6.1 \pm 1.20$ & $5.5 \pm 2.12$ & 10 \\
\hline $\begin{array}{c}\text { Sardella et al., [24] } \\
(2008) \\
\text { (H. perforatum extract) }\end{array}$ & $\begin{array}{l}0-10 \text { VAS } \\
(12 \text { weeks })\end{array}$ & $6.77 \pm 2.1 \#$ & $5 \pm 3.02 \#$ & 19 & $7.28 \pm 2.08 \#$ & $6.15 \pm 2.44 \#$ & 20 \\
\hline $\begin{array}{l}\text { Spanemberg et al., [27] } \\
\text { (2012) (catuama) }\end{array}$ & $\begin{array}{l}0-10 \mathrm{VNS} \\
(8 \text { weeks) }\end{array}$ & $6.87 \pm 2.16$ & $3.33 \pm 2.56$ & 30 & $7.17 \pm 2.0$ & $5.47 \pm 2.76$ & 30 \\
\hline $\begin{array}{l}\text { Tammiala-Salonen \& } \\
\text { Forssell,[23] (1999) } \\
\text { (trazodone) }\end{array}$ & $\begin{array}{l}0-100 \text { VAS } \\
(8 \text { weeks })\end{array}$ & $59.2 \pm 18^{*}$ & $45.3 \pm 18^{*}$ & 18 & $46.6 \pm 16^{*}$ & $34.3 \pm 28^{*}$ & 19 \\
\hline $\begin{array}{c}\text { Valenzuela et al., [28] } \\
\text { (2015) } \\
\text { (Topical chamomile) }\end{array}$ & $\begin{array}{l}0-10 \text { VAS } \\
\text { (1 month) }\end{array}$ & $7.4 \pm 8.35$ & $6.7 \pm 8.0$ & 31 & $6.9 \pm 9.18$ & $6.2 \pm 9.69$ & 26 \\
\hline
\end{tabular}

VAS = Visual Analog Scale; NRS = Numeric Rating Scale; VNS = Visual Numeric Scale.

* estimated from graph in [23].

\# estimated from table in [24]. 
The diagnosis of BMS followed in accordance to the International Classification of Headaches: "Continuous symptoms of oral burning or pain on a daily or almost daily basis, during all or part of the day for more than 6 months" [5]. The included patients were diagnosed as idiopathic or 'essential' BMS with clinical symptoms, which is burning sensation in the oral mucosa, lips, alveolar ridge and tongue, despite the absence of clinical findings and abnormalities in the laboratory testing or imaging. The patients with abnormal neurological conditions and those regularly treated on a daily basis with antidepressants, anticonvulsants, psychotropic drugs or psychological therapy were excluded in a few studies. [10, 16, 21, 23, 27, 29] Occasional use of an anxiolytic to induce sleep was accepted [10] as well as patients taking psychotropic drugs, such as anxiolytics or antidepressants [28]. The length of time that the subjects had symptoms of BMS was: 2 months $(\mathrm{n}=1), 3$ months $(\mathrm{n}=2), 4$ months $(n=4), 6$ months $(n=9)$ and six studies did not mention the time the patients had BMS. The largest sample size in the treatment group was 38 patients [27] and the smallest was nine [20]; in general the studies had very small sample size. Fourteen studies used a VAS or NRS scale to measure the primary outcome of change in pain intensity from the baseline $[8,10,14,16-19,21-24,27-29]$ (Table 3). Seven studies reported the number of patients with improvement in pain after the interventions $[8,9,12,13,15$, $16,25]$, however, there was heterogeneity in the measurement of the improvement (Table 4). Five studies [10, $18,19,22,27]$ reported the number of responders $50 \%$ or more of pain reduction (Table 5).

Table 4. Secondary outcome: Any improvement in patient's symptoms.

\begin{tabular}{|c|c|c|c|c|}
\hline Study & Outcome reported/Duration of Tx & $\begin{array}{l}\text { Treatment Group } \\
\text { N improved/Total N } \\
\text { (\% Improved) }\end{array}$ & $\begin{array}{c}\text { Placebo Group } \\
\text { Nimproved/Total N } \\
\text { (\% Improved) }\end{array}$ & $\begin{array}{r}\text { Estimated } \\
\text { Measure effects } \\
\text { (Risk Ratio) }\end{array}$ \\
\hline $\begin{array}{l}\text { Bergdahl et al.,[8] } \\
(1995)(\mathrm{CBT})\end{array}$ & $\begin{array}{l}\text { Patients symptom-free or with } \\
\text { pronounced reduction in BMS intensity. } \\
\text { Post-treatment }\end{array}$ & $\begin{array}{l}4 / 15 \\
(27 \%)\end{array}$ & $\begin{array}{l}0 / 15 \\
(0 \%)\end{array}$ & $\mathrm{RR}=9.0$ \\
\hline $\begin{array}{c}\text { Femiano \& } \\
\text { Scully,[12] (2002) (ALA) }\end{array}$ & $\begin{array}{l}\text { Patients with slight improvement, decided } \\
\text { improvement or resolution of symptoms. } \\
2 \text { mo. }\end{array}$ & $\begin{array}{l}29 / 30 \\
(97 \%)\end{array}$ & $\begin{array}{l}12 / 30 \\
(40 \%)\end{array}$ & $\mathrm{RR}=2.417$ \\
\hline $\begin{array}{l}\text { Lopez-D’Alessandro } \\
\& \text { Escovich, [13] (2011) } \\
\text { (ALA) }\end{array}$ & $\begin{array}{l}\text { Patients with positive improvements or } \\
\text { full recovery. } \\
2 \text { mo. }\end{array}$ & $\begin{array}{l}11 / 20 \\
(55 \%)\end{array}$ & $\begin{array}{l}9 / 60 \\
(15 \%)\end{array}$ & $\mathrm{RR}=3.667$ \\
\hline $\begin{array}{l}\text { Lopez-D’Alessandro } \\
\text { \& Escovich,[13] (2011) } \\
\text { (gabapentin) }\end{array}$ & $\begin{array}{l}\text { Patients with positive improvements or } \\
\text { full recovery. } \\
2 \text { mo. }\end{array}$ & $\begin{array}{l}10 / 20 \\
(50 \%)\end{array}$ & $\begin{array}{l}9 / 60 \\
(15 \%)\end{array}$ & $\mathrm{RR}=3.333$ \\
\hline $\begin{array}{l}\text { Miziara et al.,[9] } \\
(2009)(\mathrm{CBT})\end{array}$ & $\begin{array}{l}\text { Improvement in the intensity of } \\
\text { symptoms (at least of one grade) measured } \\
\text { by the PPI index. } \\
1 \text { mo. }\end{array}$ & $\begin{array}{c}17 / 24 \\
(70.8 \%)\end{array}$ & $\begin{array}{c}8 / 20 \\
(40 \%)\end{array}$ & $\mathrm{RR}=1.771$ \\
\hline $\begin{array}{l}\text { Marino et al.,[16] } \\
(2010) \text { (ALA) }\end{array}$ & $\begin{array}{l}\text { Improvement in VAS scale. } \\
2 \text { months }\end{array}$ & $\begin{array}{c}8 / 14 \\
(57 \%)\end{array}$ & $\begin{array}{l}0 / 14 \\
(0 \%)\end{array}$ & $\mathrm{RR}=17.0$ \\
\hline $\begin{array}{l}\text { Marino et al.,[16] } \\
\text { (2010) (capsaicin) }\end{array}$ & $\begin{array}{c}\text { Improvement in VAS scale. } \\
2 \text { months }\end{array}$ & $\begin{array}{l}10 / 14 \\
(76 \%)\end{array}$ & $\begin{array}{l}0 / 14 \\
(0 \%)\end{array}$ & $\mathrm{RR}=21.0$ \\
\hline $\begin{array}{l}\text { Palacios-Sanchez et } \\
\text { al.,[15] (2015) (ALA) }\end{array}$ & $\begin{array}{l}\text { Patients with slight improvement, decided } \\
\text { improvement or resolution. } \\
\qquad 2 \text { months }\end{array}$ & $\begin{array}{l}16 / 25 \\
(64 \%)\end{array}$ & $\begin{array}{c}8 / 29 \\
(27.6 \%)\end{array}$ & $\mathrm{RR}=2.320$ \\
\hline $\begin{array}{l}\text { Petruzzi et al., [25] } \\
\text { (2004) (capsaicin) }\end{array}$ & $\begin{array}{l}\text { Patients with improvement in VAS scale. } \\
\qquad 1 \mathrm{mo} .\end{array}$ & $\begin{array}{l}19 / 25 \\
(76 \%)\end{array}$ & $\begin{array}{l}1 / 25 \\
(4 \%)\end{array}$ & $\mathrm{RR}=19.0$ \\
\hline
\end{tabular}

Table 5. Number of responders, patients with a $50 \%$ or more reduction in pain intensity measured with a VAS.

\begin{tabular}{|c|c|c|c|c|c|}
\hline Study & Pain intensity scale & Duration Tx & $\begin{array}{c}\text { TREATMENT } \\
\text { GROUP } \\
\text { N responders } \\
\text { (\% responders) }\end{array}$ & $\begin{array}{c}\text { PLACEBO GROUP } \\
\text { N responders } \\
\text { (\% responders) }\end{array}$ & $\begin{array}{c}\text { Estimated Measure } \\
\text { effects } \\
\text { (Risk Ratio) }\end{array}$ \\
\hline $\begin{array}{c}\text { Carbone et al., [10] (2009) } \\
\text { (ALA) }\end{array}$ & VAS 0-10 & 8 weeks & $\begin{array}{c}4 / 22 \\
(18 \%)\end{array}$ & $\begin{array}{c}6 / 22 \\
(27.3 \%)\end{array}$ & $\mathrm{RR}=0.667$ \\
\hline $\begin{array}{c}\text { Gremeau-Richard et al., } \\
\text { [19] (2004) (topical } \\
\text { clonazepam) }\end{array}$ & VAS 0-10 & 2 weeks & $\begin{array}{c}16 / 22 \\
(72.7 \%)\end{array}$ & $\begin{array}{c}3 / 23 \\
(13.0 \%)\end{array}$ & $\mathrm{RR}=5.576$ \\
\hline $\begin{array}{c}\text { Rodriguez de } \\
\text { Rivera-Campillo et al.,[18] } \\
\text { (2010) (topical } \\
\text { clonazepam) }\end{array}$ & VAS 0-10 & 1 month & $\begin{array}{c}23 / 33 \\
(69 \%)\end{array}$ & $\begin{array}{c}4 / 33 \\
(12 \%)\end{array}$ \\
\hline $\begin{array}{c}\text { Sardella et al., [22](1999) } \\
\text { (benzydamine HCl) }\end{array}$ & VAS 0-8 & 1 mo. & $\begin{array}{c}1 / 10 \\
(10.0 \%)\end{array}$ & $\begin{array}{c}2 / 10 \\
(20.0 \%)\end{array}$ & $\mathrm{RR}=0.500$ \\
\hline $\begin{array}{c}\text { Spanemberg et al., [27] } \\
\text { (2012) (Catuama) }\end{array}$ & VAS 0-10 & 8 weeks & $52.4 \%$ & $24.2 \%$ & $\mathrm{RR}=2.165$ \\
\hline
\end{tabular}




\subsection{Risk of Bias in Included Studies}

Risk of bias summary and graph are displayed in Table 6 and Figure 2. The overall assessment of risk of bias shows only one study [28] at low risk of bias, twelve studies [10-13, 17, 18, 20, 22, 24-26, 29] were assessed at unclear risk of bias and nine studies $[8,9,14-16,19,21,23,27]$ at high risk of bias (Table 6, Figure 2).

Table 6. Summary of risk of bias for eligible studies

\begin{tabular}{|c|c|c|c|c|c|c|c|}
\hline Study & $\begin{array}{c}\text { Random Seq. } \\
\text { Generation }\end{array}$ & $\begin{array}{c}\text { Allocation } \\
\text { Concealment }\end{array}$ & Blinding & $\begin{array}{c}\text { Incomplete } \\
\text { Outcome Data }\end{array}$ & $\begin{array}{l}\text { Selective } \\
\text { Reporting }\end{array}$ & $\begin{array}{c}\text { Other } \\
\text { potential } \\
\text { bias } \\
\end{array}$ & $\begin{array}{c}\text { Overall } \\
\text { Risk of } \\
\text { Bias } \\
\end{array}$ \\
\hline Bergdahl et al.,[8] (1995) & $?$ & + & + & - & - & $?$ & + \\
\hline $\begin{array}{l}\text { Cano-Carrillo et al.,[29] } \\
\text { (2014) }\end{array}$ & - & - & - & $?$ & - & $?$ & $?$ \\
\hline Carbone et al., [10] (2009) & - & - & $?$ & - & - & $?$ & $?$ \\
\hline $\begin{array}{c}\text { Cavalcanti \& da } \\
\text { Silveira,[11] (2009) }\end{array}$ & - & - & $?$ & $?$ & - & - & $?$ \\
\hline $\begin{array}{l}\text { Femiano \& Scully,[12] } \\
\text { (2002) }\end{array}$ & $?$ & - & $?$ & - & - & $?$ & $?$ \\
\hline $\begin{array}{c}\text { Gremeau-Richard et al., } \\
{[19](2004)}\end{array}$ & - & - & $?$ & - & - & + & + \\
\hline $\begin{array}{c}\text { Gremeau-Richard et } \\
\text { al.,[21] (2010) }\end{array}$ & + & + & + & - & - & + & + \\
\hline Heckmann et al.,[17](2012) & - & - & $?$ & - & - & - & $?$ \\
\hline $\begin{array}{l}\text { Lopez-D'Alessandro \& } \\
\text { Escovich,[13] (2011) }\end{array}$ & - & - & $?$ & - & - & $?$ & $?$ \\
\hline $\begin{array}{l}\text { Lopez-Jornet et al.,[14] } \\
\text { (2009) }\end{array}$ & - & - & $?$ & + & - & $?$ & + \\
\hline Marino et al.,[16] (2010) & - & + & + & - & - & - & + \\
\hline Miziara et al.,[9] (2009) & $?$ & $?$ & + & - & - & - & + \\
\hline $\begin{array}{l}\text { Palacios-Sanchez et al.,[15] } \\
\text { (2015) }\end{array}$ & $?$ & $?$ & $?$ & + & - & $?$ & + \\
\hline Petruzzi et al., [25] (2004) & $?$ & $?$ & - & - & - & - & $?$ \\
\hline $\begin{array}{c}\text { Rodriguez de } \\
\text { Rivera-Campillo et al.,[18] } \\
(2010)\end{array}$ & - & - & $?$ & - & - & - & $?$ \\
\hline Sardella et al., [22] (1999) & - & - & $?$ & - & - & - & $?$ \\
\hline Sardella et al., [24] (2008) & - & - & $?$ & - & - & $?$ & $?$ \\
\hline Silvestre et al.,[26] (2012) & $?$ & $?$ & $?$ & - & - & - & $?$ \\
\hline $\begin{array}{c}\text { Spanemberg et al., [27] } \\
\text { (2012) }\end{array}$ & $?$ & - & $?$ & + & - & - & + \\
\hline $\begin{array}{c}\text { Tammiala-Salonen \& } \\
\text { Forssell,[23] (1999) }\end{array}$ & - & - & $?$ & + & - & + & + \\
\hline Treldal et al.,[20] (2016) & - & - & - & - & - & $?$ & $?$ \\
\hline $\begin{array}{l}\text { Valenzuela et al., [28] } \\
\text { (2015) }\end{array}$ & - & - & - & - & - & - & - \\
\hline
\end{tabular}




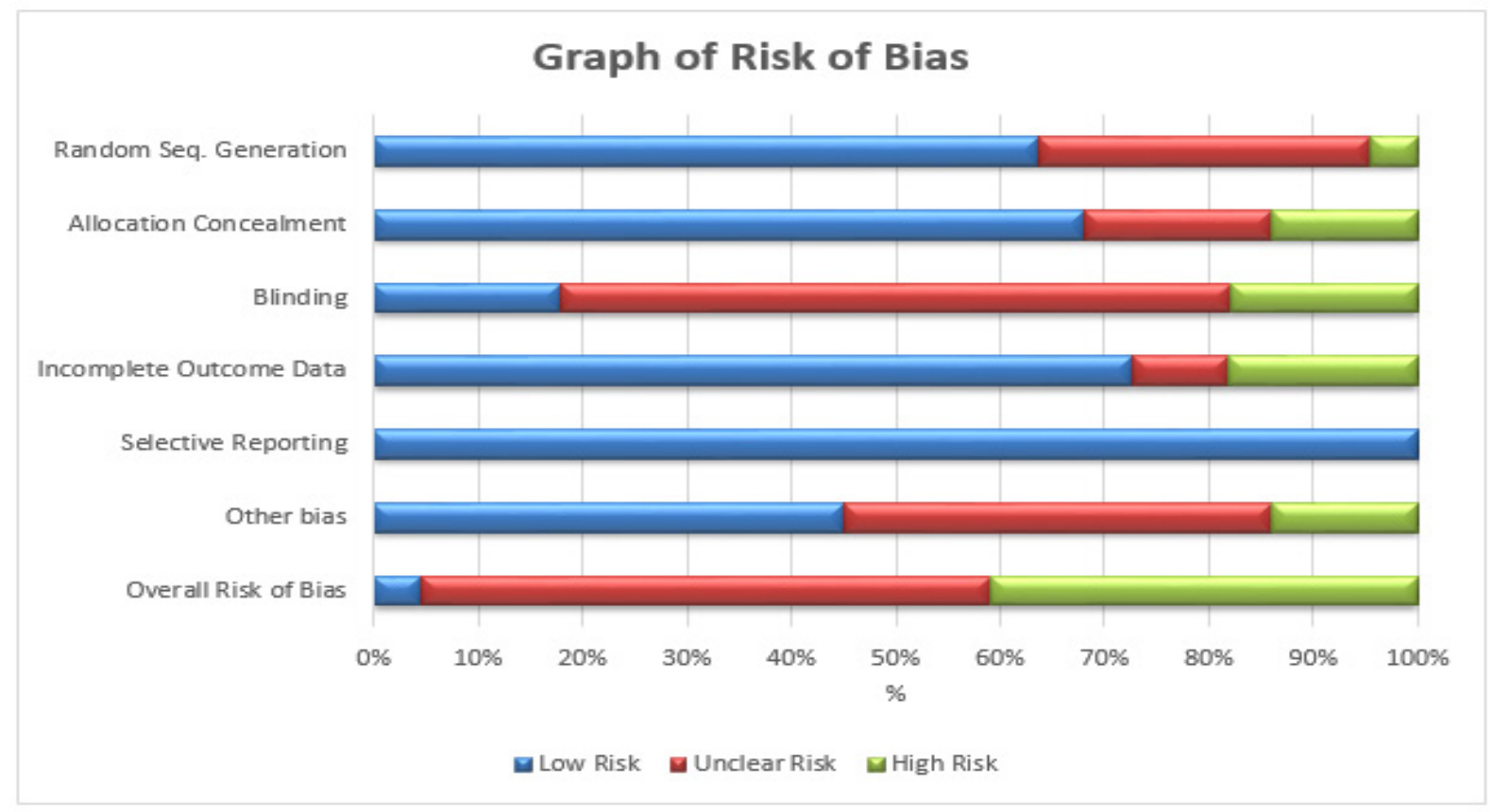

Figure 2. Risk of bias for eligible studies

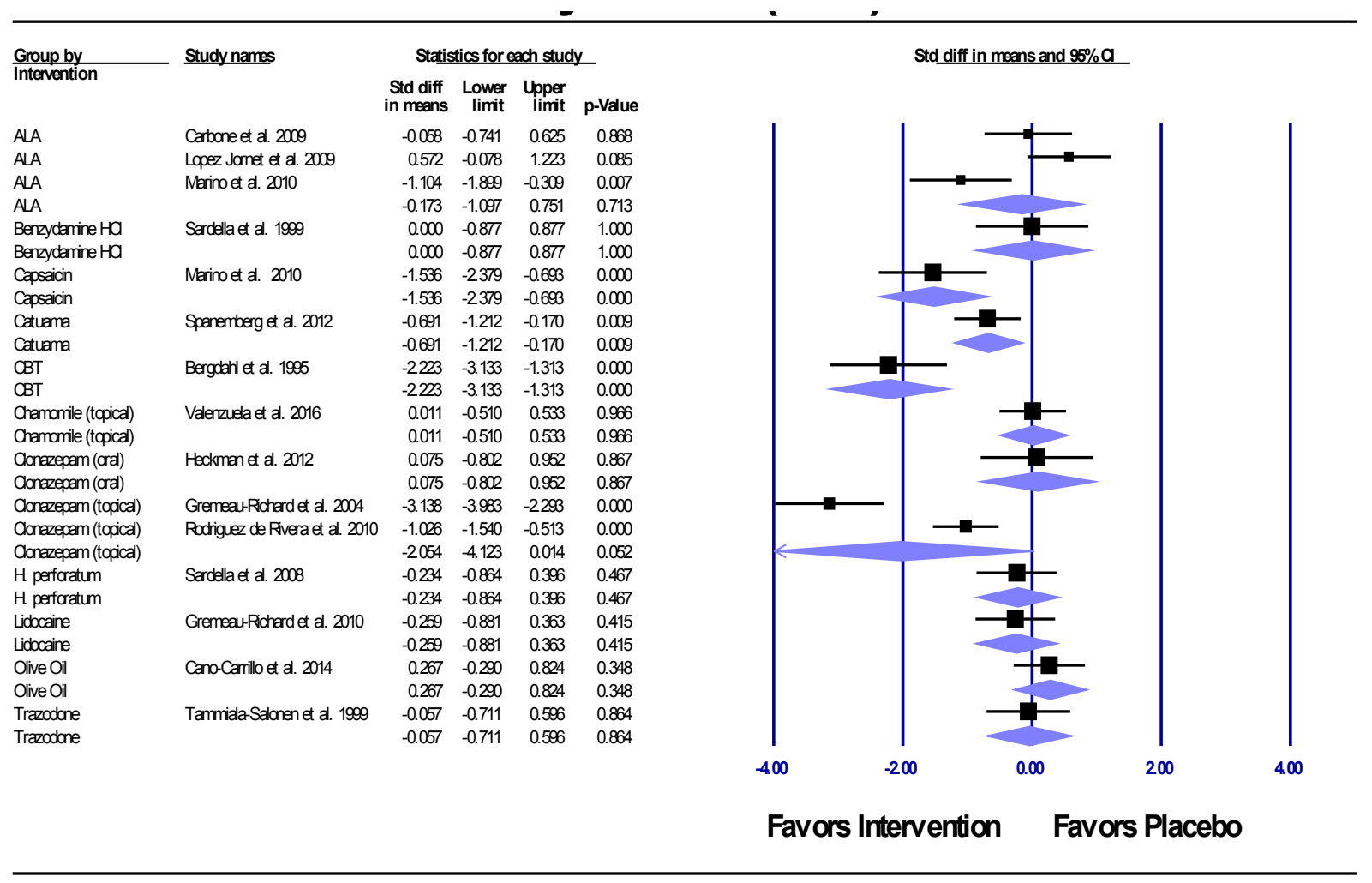

Figure 3. RCTs comparing any intervention versus placebo for the treatment of BMS. Subgroup analyses by BMS intervention reporting the change in pain intensity from baseline to after treatment.

\subsection{Effect of Interventions}

\subsubsection{Change in Pain Intensity from Baseline}

Fourteen studies reporting average and standard deviation of pain intensity at baseline and post-treatment were included in subgroup analyses (Table 3). Three studies did not provide standard deviation [11] or placebo data [20, 26] and could not be included in the meta-analysis. Due to the heterogeneity of the pain scales, we used the standardized differences in means (SDM) with $95 \%$ confidence intervals to estimate the pooled effects. Because of the heterogeneity of the interventions the overall pooled results estimates are 
not shown. However, subgroup analyses for ALA, benzydamine $\mathrm{HCl}$, capsaicin, catuama, CBT, chamomile, clonazepam (oral), clonazepam (topical), H. perforatum, lidocaine, lycopene-enriched olive oil and trazodone are shown (Figure 3).

Six studies compared ALA to placebo but only three reported means and standard deviations of pain intensity at baseline and 2 months post-treatment $[10,14,16]$. There was significant heterogeneity among ALA's studies (Q $\mathrm{p}$-value $=.006 ; \mathrm{I}^{2}=80 \%$ ). The pooled results with random effects model showed no significant reduction in pain intensity at 2 months with ALA treatment compared to placebo $(\mathrm{SDM}=-0.173,95 \% \mathrm{CI}-1.097$ to $0.751 ; \mathrm{p}=0.713)$. One study on oral clonazepam showed no significant effect in pain intensity $(\mathrm{p}=.867)$. Two studies on topical clonazepam versus placebo measured short term effects (4-6 weeks). High statistical heterogeneity was found (Q-test $\mathrm{p}<.001 ; \mathrm{I}^{2}=94 \%$ ). According to the random effects model, the pooled results showed a non-significant improvement in post-treatment pain intensity with topical clonazepam versus placebo $(\mathrm{SMD}=-2.054,95 \% \mathrm{CI}-4.123$ to 0.014 ; random $\mathrm{p}=.052$ ), however a trend toward significance was apparent. Individual studies show a significant improvement in pain with capsaicin $(\mathrm{p}<.001)$, catuama $(\mathrm{p}=.009)$ and CBT $(p<.001)$. No significant differences were found in individual studies reporting pain reduction with benzydamine $\mathrm{HCl}$, chamomile, $\mathrm{H}$. perforatum, lidocaine, lycopene-enriched olive oil and trazodone $(\mathrm{p}>.05)$ (Figure 3).

\subsubsection{Any Improvement in Patient's Symptoms}

Seven studies [8, 9, 12, 13, 15, 16, 25] reported improvement in patient's symptoms (Table 4). Due to the heterogeneity of the interventions, overall pooled results are not shown but subgroup analyses for ALA, capsaicin, CBT and gabapentin are shown (Figure 4). Statistical heterogeneity of the 4 ALA studies was zero (Q-test $p=.417$; $\mathrm{I}^{2}=0 \%$ ) and fixed and random effect models gave the same results. ALA improved patients' symptoms significantly $(\mathrm{RR}=2.676 ; 95 \% \mathrm{CI}=1.933$ to $3.705 ; \mathrm{p}<.001)$. No statistical heterogeneity was found among capsaicin studies (Q-test $\mathrm{p}=.953 ; \mathrm{I}^{2}=0 \%$ ). Capsaicin also improved patients' symptoms significantly $(\mathrm{RR}=19.641 ; 95 \% \mathrm{CI}=4.044$ to 95.402; $<<.001)$. Statistical heterogeneity of the CBT versus placebo studies was low and not significant (Q-test $\mathrm{p}=.272$; $\mathrm{I}^{2}=17 \%$ ) and fixed effect model was used. CBT improved patients' symptoms significantly compared to placebo $(\mathrm{RR}=1.896 ; 95 \% \mathrm{CI}=1.059$ to $3.395 ; \mathrm{p}=.031)$. One study comparing gabapentin to placebo also found significant improvement in patients' symptoms $(\mathrm{RR}=3.333$; $95 \% \mathrm{CI}=$ 1.583 to $7.021 ; \mathrm{p}=.002)$. Due to the heterogeneity in the definition of improvement in symptoms, these results should be taken with caution (Figure 4).

\subsubsection{Number of Responders (Patients with 50\% Reduction in Pain Intensity)}

Five studies reported the number of patients with at least a $50 \%$ reduction in pain intensity using a visual analog scale or visual numeric scale $[10,18,19,22,27]$ (Table 5) Due to the heterogeneity of the interventions, overall pooled results are not shown but subgroup analyses for ALA, benzydamine $\mathrm{HCl}$, catuama, and clonazepam (topical) are shown for visual purposes (Figure 5). Statistical heterogeneity for studies of ALA versus placebo was found (Q-test $\mathrm{p}=.004 ; \mathrm{I}^{2}=88 \%$ ) and random-effects model was used. The number of responders was not statistically significantly higher with ALA than placebo $(\mathrm{p}=.520)$, nor benzydamine $\mathrm{HCl}(\mathrm{p}=.543)$. However, results with only one study showed a significant higher number of responders to treatment with catuama $(\mathrm{p}=.035)$ and topical clonazepam $(\mathrm{p}=.002)$ compared to placebo (Figure 5).

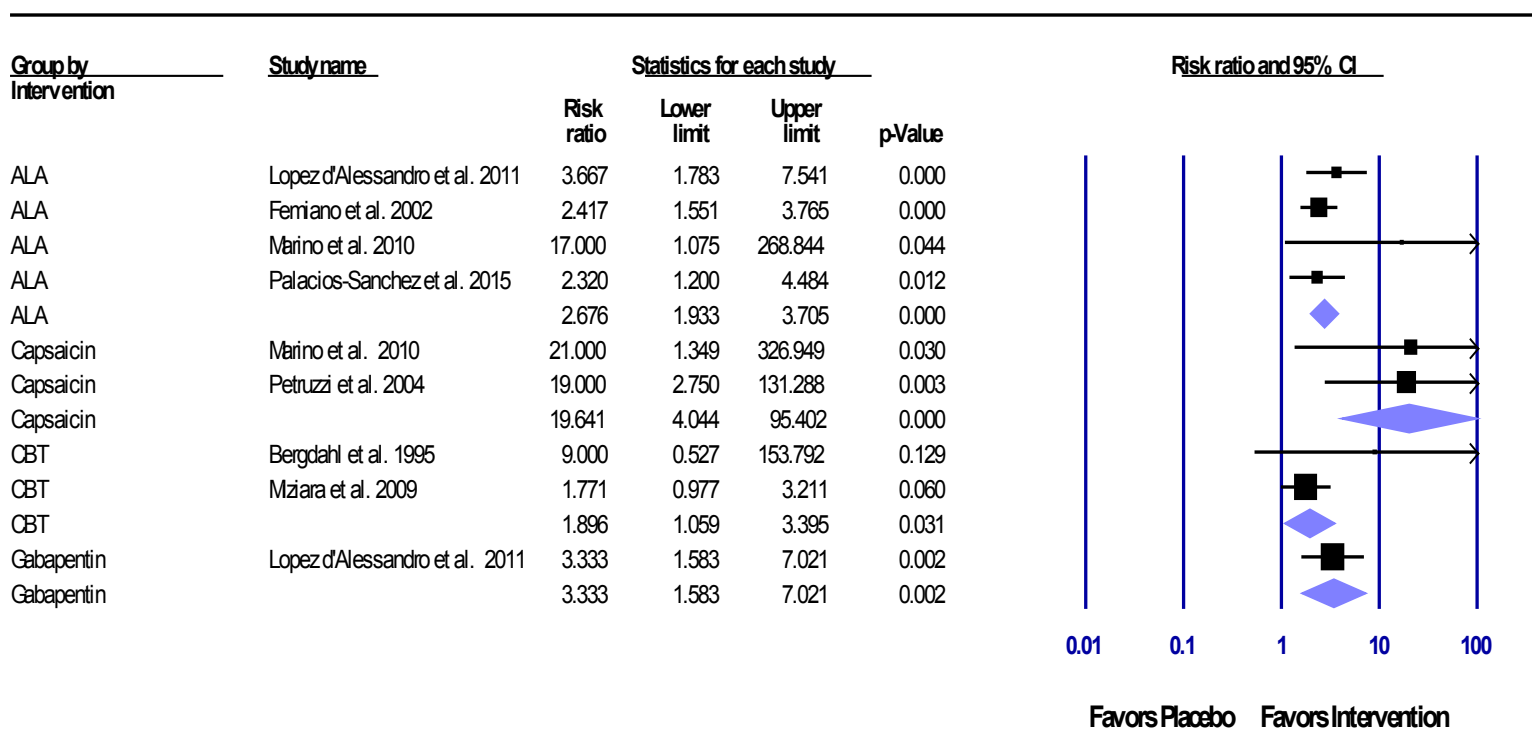

Figure 4. RCTs comparing any intervention versus placebo for the treatment of BMS. Subgroup analyses by BMS intervention reporting patients with any improvement in patients' symptoms. 


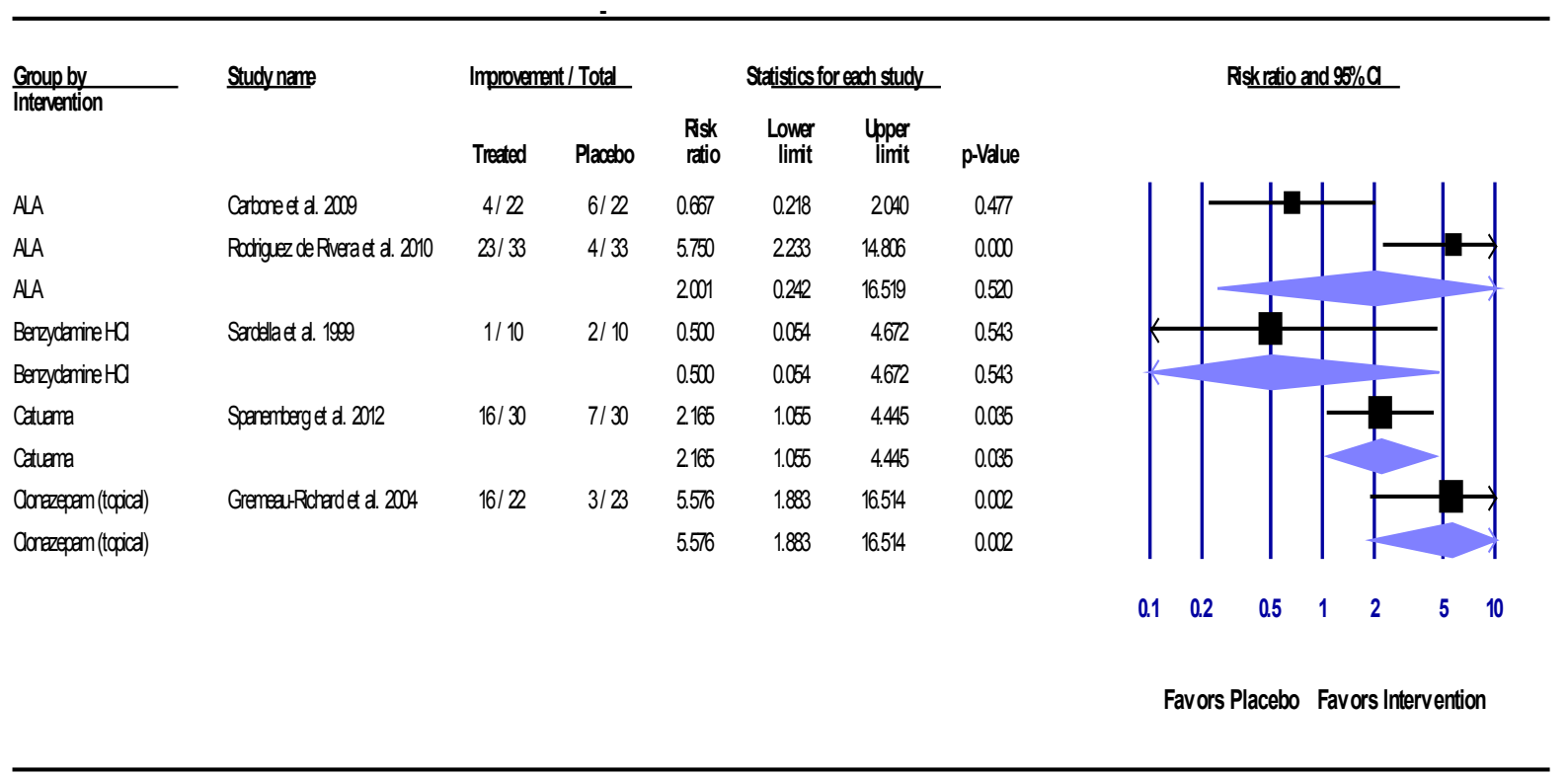

Figure 5. Subgroup analyses by BMS intervention reporting the number of responders

\section{Discussion}

Currently employed therapies (benzodiazepines, anticonvulsants, antidepressants, capsaicin, analgesics, alpha-lipoic acid, cognitive behavioral therapies and nutritional supplements) for the treatment of BMS were investigated in this systematic review. Given the heterogeneity of the interventions and outcomes reported, review authors could not provide treatment efficacy for all the interventions under study. Pooled results show that ALA through systemic delivery is not significantly effective in reducing pain as compared to placebo after 2 months of treatment $(p=.713)$. The use of topical clonazepam to decrease pain as compared to placebo showed a trend towards significance $(p=.052)$, but not when oral systemic clonazepam $(p=.867)$ was used. Nevertheless, according to our subgroup meta-analyses, ALA through systemic delivery $(\mathrm{p}<.001)$, capsaicin $(\mathrm{p}<.001)$, CBT $(\mathrm{p}=.031)$ and gabapentin $(p=.002)$ were significantly effective in improving patient's symptoms compared to placebo at 1-2 months of treatment. However, due to the heterogeneity of the definition of improvement used by the authors to measure patient's improvement in symptoms and the small number of studies reporting this outcome, these results should be taken with caution.

Three reviewers independently assessed the risk of bias according to the guidelines of the Cochrane Reviewers' Handbook [38]. Only randomized controlled studies with a placebo group were included in this review. The overall assessment of risk of bias showed only one study at low risk of bias [28] (Table 6). The quality of the evidence based on GRADE summary tables was moderate to low due to the small number of studies in each subgroup analysis, statistical heterogeneity and unclear or high risk of bias (Table 6). A limitation of this review however was the lack of access to EMBASE (an international biomedical database for biomedical researchers) at our institution. Some studies could have been missed due to this fact, however all included studies and reviews were hand-searched for additional studies. This review's results apply to adult patients diagnosed with primary BMS. 
Table 6. Quality of the evidence (GRADE) and summary of findings table

\begin{tabular}{|c|c|c|c|c|c|}
\hline \multicolumn{6}{|c|}{ ALA compared to placebo for BMS } \\
\hline \multirow[t]{2}{*}{ Outcomes } & \multirow{2}{*}{$\begin{array}{l}\text { No of } \\
\text { Participants } \\
\text { (studies) } \\
\text { Follow up }\end{array}$} & \multirow{2}{*}{\multicolumn{2}{|c|}{$\begin{array}{l}\text { Quality of the evidence:Relative } \\
\begin{array}{l}\text { (GRADE) } \\
\text { (effect }\end{array}\end{array}$}} & \multicolumn{2}{|c|}{ Anticipated absolute effects } \\
\hline & & & & $\begin{array}{l}\text { Risk with } \\
\text { Placebo }\end{array}$ & $\begin{array}{l}\text { Risk difference with ALA ( } 95 \% \\
\text { CI) }\end{array}$ \\
\hline $\begin{array}{l}\text { Intensity of pain } \\
\text { VAS. Scale from: } 0 \text { to } 10 .\end{array}$ & $\begin{array}{l}101 \\
(3 \text { studies }) \\
2 \text { months }\end{array}$ & $\begin{array}{l}\oplus \oplus \ominus \ominus \\
\text { LOW } \\
\text { due to risk of bias, } \\
\text { inconsistency }\end{array}$ & & & $\begin{array}{l}\text { The mean intensity of pain in the } \\
\text { intervention groups was } \\
0.085 \text { standard deviations lower } \\
(0.49 \text { lower to } 0.32 \text { higher })\end{array}$ \\
\hline \multirow{4}{*}{$\begin{array}{l}\text { Improvement in patient's } \\
\text { symptoms } \\
\text { scale }\end{array}$} & \multirow{4}{*}{$\begin{array}{l}232 \\
(4 \text { studies }) \\
2 \text { months }\end{array}$} & \multirow{4}{*}{$\begin{array}{l}\oplus \oplus \oplus \ominus \\
\text { MODERATE } \\
\text { due to risk of bias }\end{array}$} & \multirow{4}{*}{$\begin{array}{l}\text { RR 2.676 } \\
(1.933 \text { to } \\
3.705)\end{array}$} & \multicolumn{2}{|c|}{ Study population } \\
\hline & & & & \multicolumn{2}{|c|}{\begin{tabular}{|r|l|}
203 per 1000 & 340 more per 1000 \\
(from 189 more to 549 more)
\end{tabular}} \\
\hline & & & & \multicolumn{2}{|l|}{ Moderate } \\
\hline & & & & & - \\
\hline \multirow[t]{4}{*}{ Number of responders } & \multirow{4}{*}{$\begin{array}{l}107 \\
(2 \text { studies }) \\
1-2 \text { months }\end{array}$} & \multirow{4}{*}{$\begin{array}{l}\oplus \oplus \Theta \ominus \\
\text { LOW } \\
\text { due to risk of bias, } \\
\text { inconsistency }\end{array}$} & \multirow{4}{*}{$\begin{array}{l}\text { RR 2.001 } \\
(0.242 \text { to } \\
16.519)\end{array}$} & \multicolumn{2}{|c|}{ Study population } \\
\hline & & & & \multicolumn{2}{|c|}{\begin{tabular}{|l|l}
192 per 1000 & 192 more per 1000 \\
(from 146 fewer to 1000 more)
\end{tabular}} \\
\hline & & & & \multicolumn{2}{|c|}{ Moderate } \\
\hline & & & & & - \\
\hline
\end{tabular}

\begin{tabular}{|c|c|c|c|c|c|c|c|}
\hline \multicolumn{8}{|c|}{ Clonazepam (topical) compared to placebo for treatment of BMS } \\
\hline \multirow[t]{2}{*}{ Outcomes } & \multirow{2}{*}{$\begin{array}{l}\text { No of } \\
\text { Participants } \\
\text { (studies) } \\
\text { Follow up } \\
\end{array}$} & \multirow{2}{*}{\multicolumn{2}{|c|}{$\begin{array}{l}\text { Quality of the evidence } \\
\text { (GRADE) }\end{array}$}} & \multirow{2}{*}{$\begin{array}{l}\text { Relative } \\
\text { effect } \\
(95 \% \text { CI) }\end{array}$} & \multicolumn{3}{|c|}{ Anticipated absolute effects } \\
\hline & & & & & $\begin{array}{l}\text { Risk with } \\
\text { Placebo }\end{array}$ & \multicolumn{2}{|c|}{$\begin{array}{l}\text { Risk difference with Clonazepam } \\
\text { (topical) }(95 \% \mathrm{CI})\end{array}$} \\
\hline $\begin{array}{l}\text { Intensity of } \\
\text { pain } \\
\text { Scale } 0-10\end{array}$ & $\begin{array}{l}114 \\
(2 \text { studies }) \\
2-4 \text { weeks }\end{array}$ & \multicolumn{2}{|c|}{$\begin{array}{l}\oplus \oplus \ominus \ominus \\
\text { LOW }{ }^{1,2} \\
\text { due to risk of bias, } \\
\text { inconsistency }\end{array}$} & & & \multicolumn{2}{|c|}{$\begin{array}{l}\text { The mean intensity of pain in the } \\
\text { intervention groups was } \\
\mathbf{2 . 0 5 4} \text { standard deviations lower } \\
\text { (4.123 lower to } 0.014 \text { higher) }\end{array}$} \\
\hline \multicolumn{8}{|c|}{$\begin{array}{l}\text { All included studies at unclear or high risk of bias } \\
\text { Inconsistency due to variation of the effect and significant heterogeneity (Q p-value }<.05 ; \mathrm{I} 2>=90 \%)\end{array}$} \\
\hline \multicolumn{8}{|c|}{ Capsaicin compared to placebo for treatment of BMS } \\
\hline \multirow{2}{*}{\multicolumn{2}{|c|}{ Outcomes }} & \multirow{2}{*}{$\begin{array}{l}\text { No of } \\
\text { Participants } \\
\text { (studies) } \\
\text { Follow up }\end{array}$} & \multirow{2}{*}{\multicolumn{2}{|c|}{$\begin{array}{l}\text { Quality of the } \\
\text { evidence } \\
\text { (GRADE) }\end{array}$}} & \multirow{2}{*}{$\begin{array}{l}\text { Relative } \\
\text { effect } \\
(95 \% \mathrm{CI})\end{array}$} & \multicolumn{2}{|c|}{ Anticipated absolute effects } \\
\hline & & & & & & $\begin{array}{l}\text { Risk with } \\
\text { Placebo }\end{array}$ & $\begin{array}{l}\text { Risk difference with } \\
\text { Capsaicin }(95 \% \mathrm{CI})\end{array}$ \\
\hline \multicolumn{2}{|c|}{$\begin{array}{l}\text { Improvement in patient's } \\
\text { symptoms } \\
\text { scale }\end{array}$} & $\begin{array}{l}78 \\
(2 \text { studies }) \\
1-2 \text { months }\end{array}$ & \multicolumn{2}{|c|}{$\begin{array}{l}\oplus \oplus \Theta \ominus \\
\text { LOW } \\
\text { due to risk of bias, } \\
\text { imprecision }\end{array}$} & $\begin{array}{l}\text { RR } 19.641 \\
(4.044 \text { to } \\
95.402)\end{array}$ & 26 per 1000 & $\begin{array}{l}478 \text { more per } 1000 \\
\text { (from } 78 \text { more to } 1000 \text { more) }\end{array}$ \\
\hline
\end{tabular}




\begin{tabular}{|c|c|c|c|c|c|}
\hline \multicolumn{6}{|c|}{ CBT compared to placebo group for treatment of BMS } \\
\hline Outcomes & $\begin{array}{l}\text { No of } \\
\text { Participants } \\
\text { (studies) } \\
\text { Follow up }\end{array}$ & $\begin{array}{l}\text { Quality of the } \\
\text { evidence } \\
\text { (GRADE) }\end{array}$ & $\begin{array}{l}\text { Relative } \\
\text { effect } \\
(95 \% \text { CI) }\end{array}$ & $\begin{array}{l}\text { Anticipated absolu } \\
\text { Risk with Placebo } \\
\text { group }\end{array}$ & $\begin{array}{l}\text { Risk difference with CBT } \\
(95 \% \mathrm{CI})\end{array}$ \\
\hline $\begin{array}{l}\text { Improvement in patient's } \\
\text { symptoms } \\
\text { scale }\end{array}$ & $\begin{array}{l}74 \\
(2 \text { studies })\end{array}$ & $\begin{array}{l}\oplus \oplus \Theta \ominus \\
L O W^{1,2} \\
\text { due to risk of bias, } \\
\text { imprecision }\end{array}$ & $\begin{array}{l}\text { RR } 1.896 \\
(1.059 \text { to } \\
3.395)\end{array}$ & 229 per 1000 & $\begin{array}{l}\mathbf{2 0 5} \text { more per } 1000 \\
\text { (from } 13 \text { more to } 547 \\
\text { more) }\end{array}$ \\
\hline \multicolumn{6}{|c|}{$\begin{array}{l}\text { All included studies at unclear and high risk of bias } \\
2 \text { Small number of included studies }(n=2) \text { with small sample size }\end{array}$} \\
\hline \multicolumn{6}{|c|}{ CI: Confidence interval; RR: Risk ratio; } \\
\hline \multicolumn{6}{|c|}{$\begin{array}{l}\text { GRADE Working Group grades of evidence } \\
\text { High quality: Further research is very unlikely to change our confidence in the estimate of effect. } \\
\text { Moderate quality: Further research is likely to have an important impact on our confidence in the estimate of effect and may change } \\
\text { the estimate. } \\
\text { Low quality: Further research is very likely to have an important impact on our confidence in the estimate of effect and is likely to } \\
\text { change the estimate. } \\
\text { Very low quality: We are very uncertain about the estimate. }\end{array}$} \\
\hline
\end{tabular}

\subsection{Agreements and Disagreements with Other Studies}

ALA: Regarding the use of ALA for the treatment of BMS, the evidence is inconclusive. Though a non-significant reduction of pain intensity after the administration of ALA was found $(p=.713)$ in three trials $[10,14,16]$, pooled results showed a significant improvement in patient's symptoms with ALA compared to placebo $(p<.001)$. These results are consistent with reviews by De Moraes et al. [41], Kisely et al. [42] and McMillian et al. [43].

Clonazepam: In this review, two studies [18-19] showed a significant improvement in patients' symptoms with the use of topical clonazepam. In the study by Gremeau-Richard et al. [19], the use of topical clonazepam for 14 days showed $72.7 \%$ of the patients responded to treatment as compared to $13 \%$ of the placebo group. In the other study, a controlled and double blind study, $69 \%$ patients improved at least $50 \%$ in the intervention group and the authors suggested that "clonazepam acts locally, affecting one or more of the etiopathogenic mechanisms responsible for BMS" [18]. However, in a third study, the use of systemic clonazepam though it showed a significant improvement in pain ratings in the treated group $(p<.001)$ [17], the difference with the placebo group was not significant. Our study corroborates the findings by Sun et al. [44] and Gremeau-Richard et al. [19] that topical use of clonazepam is more effective than its systemic use. Concerns with clonazepam include dizziness and drowsiness (particularly in the older adult population), as well as potential withdrawal effect and physical and psychological dependence effects.

Capsaicin: Two trials were included in this review $[16,25]$ in order to assess the efficacy of topical rinse with capsaicin. Both found a significant decrease in pain ratings using the rinse, corresponding to the conclusions of two reviews [41, 42]. One more study [26] focused on capsaicin and its effect on BMS was omitted from our meta-analysis due to missing data. Intraoral topical administration of capsaicin has not been associated with significant gastric pain, unlike systemic administration of the drug [45].

CBT: Concerning the use of psychotherapy, the current review and Kisely et al. [42] showed a significant improvement in patient's symptoms, however, only two studies were available to conduct the meta-analyses $[8,9]$. Our review agrees with two other reviews that suggested that the data supporting the effects of psychotherapy was insufficient [43] and there is a lack of evidence on the efficacy of the procedure [46].

Dietary supplements and other interventions: Research on neuropathic pain has also explored the potential benefits of dietary supplements, including hypericum perforatum or St. John's Wort, [24] catuama, [27], chamomile [28] and lycopene-enriched virgin olive oil [28]. Prior reviews found no significant differences in pain improvement between placebo and St. John's Wort [42] nor chamomile or olive oil [42, 43]. Spanemberg et al. [27] reported that catuama showed a significant difference in symptom alleviation compared to placebo, supported by two reviews [42, 43]. Furthermore, Kisely et al. [42] reported the improvement in the catuama group was significantly greater in follow-ups after 4, 8, and 12 weeks.

Anesthetics, NSAIDs and antidepressants: Two topical anesthetics were analyzed in this review, bupivacaine [20] 
and lidocaine [21]. The data reported by Treldal's trial [20] was incomplete, so its results were not included in the meta-analyses. Based on our analysis of Gremeau-Richard et al. [21], lidocaine lingual nerve block did not yield any significant difference compared to placebo, in agreement with a prior review [42]. Sardella et al. [22] failed to find a significant difference with NSAID's (benzydamine $\mathrm{HCl}$ ) use versus placebo as only 1 out of 10 patients reported pain reduction greater than $50 \%$ with benzydamine $\mathrm{HCL}$ and $2 / 10$ with placebo. These results corroborated the findings of two reviews $[42,43]$. The evaluation of the antidepressant intervention relied on the results of a single trial, which found no significant improvement in BMS symptoms given the antidepressant trazadone [23] as shown in [42, 43].

\section{Conclusions}

- Over 1.3 million Americans suffer from BMS which may affect their quality of life, therefore multiple interventions have been proposed to treat BMS but the evidence to support them was low.

- Though ALA showed significant improvements in patient's subjective symptoms, there was no overall significant decrease in pain intensity.

- Treatments designed to help people to cope with the discomfort of BMS such as CBT, topical clonazepam, catuama and capsaicin may be beneficial for short periods of time though the evidence is still insufficient.

- Due to the heterogeneity of the interventions and the high risk of bias of some studies, the evidence was low. Where subgroup analysis was possible this included only a small number of trials.

- There is a need to standardize diagnostic criteria and the measurement of pain, and then further guidelines for usage of medications, dietary supplements or CBT can be proposed.

\section{Acknowledgements}

Authors acknowledge Dr. Margarita Zeichner-David for help with editing and formatting of the manuscript.

\section{REFERENCES}

[1] G. E. Gurvits and A. Tan, "Burning mouth syndrome.," World J. Gastroenterol., vol. 19, no. 5, pp. 665-72, Feb. 2013.

[2] N. Treister and S. B. Woo, "Burning Mouth Syndrome," American Academy of Oral Medicine, 2015. Available online: http://www.aaom.com/index.php?option=com content\&view $=$ article $\&$ id $=81$ :burning-mouth-syndrome $\&$ catid $=22$ :patient-c ondition-information \&Itemid $=120$.

[3] A. Scala, L. Checchi, M. Montevecchi, and I. Marini, "Update on burning mouth syndrome: Overview and patientmanagement," Crit. Rev. ORAL Biol. Med., vol. 14, no. 4, pp. 275-291, Jul. 2003.

[4] M.-P. Minguez-Sanz, C. Salort-Llorca, and F.-J. Silvestre-Donat, "Etiology of burning mouth syndrome: a review and update.," Med. Oral Patol. Oral Cir. Bucal, vol. 16, no. 2, pp. e144-e148, 2011.

[5] J. M. Zakrzewska, a M. Glenny, and H. Forssell, "Interventions for the treatment of burning mouth syndrome.," Cochrane database Syst. Rev., no. 1, p. CD002779, 2005.

[6] G. Lauria, A. Majorana, M. Borgna, R. Lombardi, P. Penza, A. Padovani, and P. Sapelli, "Trigeminal small-fiber sensory neuropathy causes burning mouth syndrome," Pain, vol. 115, no. 3, pp. 332-337, 2005.

[7] L. L. Patton, M. A. Siegel, R. Benoliel, and A. De Laat, "Management of burning mouth syndrome: systematic review and management recommendations.," Oral Surg. Oral Med. Oral Pathol. Oral Radiol. Endod., vol. 103 Suppl, p. S39.e1-13, Mar. 2007.

[8] J. Bergdahl, G. Anneroth, and H. Perris, "Cognitive therapy in the treatment of patients with resistant burning mouth syndrome: a controlled study.," J. Oral Pathol. Med., vol. 24, no. 5, pp. 213-5, May 1995.

[9] I. D. Miziara, B. C. A. Filho, R. Oliveira, and R. M. Rodrigues dos Santos, "Group psychotherapy: an additional approach to burning mouth syndrome.," J. Psychosom. Res., vol. 67, no. 5, pp. 443-8, Nov. 2009 .

[10] M. Carbone, M. Pentenero, M. Carrozzo, A. Ippolito, and S. Gandolfo, "Lack of efficacy of alpha-lipoic acid in burning mouth syndrome: a double-blind, randomized, placebo-controlled study.," Eur. J. Pain, vol. 13, no. 5, pp. 492-6, May 2009.

[11] D. R. Cavalcanti and F. R. X. da Silveira, "Alpha lipoic acid in burning mouth syndrome--a randomized double-blind placebo-controlled trial.," J. Oral Pathol. Med., vol. 38, no. 3, pp. 254-61, Mar. 2009.

[12] F. Femiano and C. Scully, "Burning mouth syndrome (BMS): double blind controlled study of alpha-lipoic acid (thioctic acid) therapy," J. Oral Pathol. Med., vol. 31, no. 5, pp. 267$269,2002$.

[13] E. López-D'alessandro and L. Escovich, "Combination of alpha lipoic acid and gabapentin, its efficacy in the treatment of Burning Mouth Syndrome: a randomized, double-blind, placebo controlled trial.," Med. Oral Patol. Oral Cir. Bucal, vol. 16, no. 5, pp. e635-40, 2011.

[14] P. López-Jornet, F. Camacho-Alonso, and S. Leon-Espinosa, "Efficacy of alpha lipoic acid in burning mouth syndrome: a randomized, placebo-treatment study.," J. Oral Rehabil., vol. 36, no. 1, pp. 52-7, Jan. 2009.

[15] B. Palacios-Sanchez, L.-A. Moreno-Lopez, R. Cerero-Lapiedra, S. Llamas-Martinez, and G. Esparza-Gomez, "Alpha lipoic acid efficacy in burning mouth syndrome. A controlled clinical trial," Med. ORAL Patol. ORAL Y Cir. $B U C A L$, vol. 20, no. 4, pp. E435-E440, Jul. 2015.

[16] R. Marino, S. Torretta, P. Capaccio, L. Pignataro, and F. Spadari, "Different therapeutic strategies for burning mouth syndrome: preliminary data.," J. Oral Pathol. Med., vol. 39, no. 8, pp. 611-6, Sep. 2010. 
[17] S. M. Heckmann, E. Kirchner, M. Grushka, M. G. Wichmann, and T. Hummel, "A double-blind study on clonazepam in patients with burning mouth syndrome," Laryngoscope, vol. 122, no. 4, pp. 813-816, 2012.

[18] E. Rodríguez de Rivera Campillo, J. López-López, and E. Chimenos-Küstner, "Response to topical clonazepam in patients with burning mouth syndrome: a clinical study.," Bull. du Groupèment Int. pour la Rech. Sci. en Stomatol. Odontol., vol. 49, no. 1, pp. 19-29, Jan. 2010.

[19] C. Gremeau-Richard, A. Woda, M. L. Navez, N. Attal, D. Bouhassira, M. C. Gagnieu, J. F. Laluque, P. Picard, P. Pionchon, and S. Tubert, "Topical clonazepam in stomatodynia: A randomised placebo-controlled study," Pain, vol. 108, no. 1-2, pp. 51-57, 2004.

[20] C. Treldal, C. B. Jacobsen, S. Mogensen, M. Rasmussen, J. Jacobsen, J. Petersen, A. M. L. Pedersen, and O. Andersen, "Effect of a local anesthetic lozenge in relief of symptoms in burning mouth syndrome," ORAL Dis., vol. 22, no. 2, pp. 123 131, Mar. 2016.

[21] C. Grémeau-Richard, C. Dubray, B. Aublet-Cuvelier, S. Ughetto, and A. Woda, "Effect of lingual nerve block on burning mouth syndrome (stomatodynia): a randomized crossover trial.," Pain, vol. 149, no. 1, pp. 27-32, Apr. 2010.

[22] A. Sardella, D. Uglietti, F. Demarosi, G. Lodi, C. Bez, and A. Carrassi, "Benzydamine hydrochloride oral rinses in management of burning mouth syndrome. A clinical trial.," Oral Surg. Oral Med. Oral Pathol. Oral Radiol. Endod., vol. 88, no. 6, pp. 683-6, Dec. 1999.

[23] T. Tammiala-Salonen and H. Forssell, "Trazodone in burning mouth pain: a placebo-controlled, double-blind study.," $J$. Orofac. Pain, vol. 13, no. 2, pp. 83-8, 1999.

[24] A. Sardella, G. Lodi, F. Demarosi, M. Tarozzi, L. Canegallo, and A. Carrassi, "Hypericum perforatum extract in burning mouth syndrome: a randomized placebo-controlled study.," $J$. Oral Pathol. Med., vol. 37, no. 7, pp. 395-401, Aug. 2008.

[25] M. Petruzzi, D. Lauritano, M. De Benedittis, M. Baldoni, and R. Serpico, "Systemic capsaicin for burning mouth syndrome: short-term results of a pilot study.," J. Oral Pathol. Med., vol. 33, no. 2, pp. 111-4, Feb. 2004

[26] F. J. Silvestre, J. Silvestre-Rangil, C. Tamarit-Santafé, and D. Bautista, "Application of a capsaicin rinse in the treatment of burning mouth syndrome," Med. Oral Patol. Oral Cir. Bucal, vol. 17, no. 1, 2012.

[27] J. Spanemberg, K. Cherubini, M. de Figueiredo, A. Gomes, M. Campos, and F. Salum, "Effect of an herbal compound for treatment of burning mouth syndrome: randomized, controlled, double-blind clinical trial.," Oral Surg. Oral Med. Oral Pathol. Oral Radiol., vol. 113, no. 3, pp. 373-7, Mar. 2012.

[28] S. Valenzuela, A. Pons-Fuster, and P. López-Jornet, "Effect of a $2 \%$ topical chamomile application for treating burning mouth syndrome: a controlled clinical trial," J. Oral Pathol. Med., p. n/a-n/a, 2015.

[29] P. Cano-Carrillo, A. Pons-Fuster, and P. López-Jornet, "Efficacy of lycopene-enriched virgin olive oil for treating burning mouth syndrome: a double-blind randomised.," $J$. Oral Rehabil., vol. 41, no. 4, pp. 296-305, Apr. 2014.

[30] E. Maglione, C. Marrese, E. Migliaro, F. Marcuccio, C. Panico, C. Salvati, G. Citro, M. Quercio, F. Roncagliolo, C. Torello, and M. Brufani, "Increasing bioavailability of (R)-alpha-lipoic acid to boost antioxidant activity in the treatment of neuropathic pain.," Acta Biomed., vol. 86, no. 3, pp. 226-33, 2015 .

[31] A. R. Gammaitoni and M. W. Davis, "Pharmacokinetics and tolerability of lidocaine patch 5\% with extended dosing," Ann. Pharmacother., vol. 36, no. 2, pp. 236-240, 2002.

[32] A. R. Gammaitoni, N. A. Alvarez, and B. S. Galer, "Safety and tolerability of the lidocaine patch $5 \%$, a targeted peripheral analgesic: a review of the literature.," J. Clin. Pharmacol., vol. 43, no. 2, pp. 111-7, 2003

[33] de L. L. C. A. de Moraes M, do Amaral Bezerra BA, da Rocha Neto PC, de Olivde Moraes M, do Amaral Bezerra BA, da Rocha Neto PC, de Oliveira Soares AC, Pinto LF, "Randomized Trials for the Treatment of Burning Mouth Syndrome: An Evidence-Based Review of the Literature," Oral Surg. Oral Med. Oral Pathol. Oral Radiol., vol. 117, no. 2, p. e221, 2014.

[34] J. A. Blumenthal, "Relaxation therapy, biofeedback, and behavioral medicine," Psychother. Theory, Res. Pract. Train., vol. 22, no. 3, pp. 516-530, 1985.

[35] D. D. Price, P. A. McGrath, A. Rafii, and B. Buckingham, "The validation of visual analogue scales as ratio scale measures for chronic and experimental pain," Pain, vol. 17, no. 1, pp. $45-56,1983$.

[36] D. D. Price, F. M. Bush, S. Long, and S. W. Harkins, "A comparison of pain measurement characteristics of mechanical visual analogue and simple numerical rating scales," Pain, vol. 56, no. 2, pp. 217-226, 1994.

[37] D. Moher, A. Liberati, J. Tetzlaff, and D. G. Altman, "Preferred reporting items for systematic reviews and meta-analyses: the PRISMA statement.," PLoS Med., vol. 6, no. 7, p. e1000097, Jul. 2009.

[38] J. Higgins and S. (editors) Green, "Cochrane Handbook for Systematic Reviews of Interventions Version 5.1.0 [updated March 2011].," 2011.

[39] W. Cochran, "The combination of estimated from different experiments.," Biomimetrics, vol. 10, pp. 101-129, 1954.

[40] J. Higgins and S. Thompson, "Quantifying heterogeneity in a meta-analysis,” Stat. Med., vol. 21, pp. 1539-1558, 2002.

[41] M. de Moraes, B. A. do Amaral Bezerra, P. C. da Rocha Neto, A. C. A. de Oliveira Soares, L. P. Pinto, A. de Lisboa Lopes Costa, M. Moraes, B. B. A. do Amaral, N. P. C. Rocha, S. A. C. Oliveira, L. P. Pinto, and C. A. L. Lopes, "Randomized trials for the treatment of burning mouth syndrome: an evidence-based review of the literature.," J. Oral Pathol. Med., vol. 41, no. 4, pp. 281-7, Apr. 2012

[42] S. Kisely, M. Forbes, E. Sawyer, E. Black, and R. Lalloo, “A systematic review of randomized trials for the treatment of burning mouth syndrome," J. Psychosom. Res., vol. 86, pp. $39-46,2016$

[43] Z. J. McMillan R, Forssell H, Buchanan JA, Glenny AM, Weldon JC, "Interventions for treating burning mouth syndrome.," Cochrane Database Syst Rev, 2016.

[44] A. Sun, K.-M. Wu, Y.-P. Wang, H.-P. Lin, H.-M. Chen, and C.-P. Chiang, "Burning mouth syndrome: a review and update," J Oral Pathol Med, vol. 42, pp. 649-655, 2013. 
[45] Y. S. Lee, H. S. Kho, Y. K. Kim, and S. C. Chung, "Influence of topical capsaicin on facial sensitivity in response to experimental pain," J. Oral Rehabil., vol. 34, no. 1, pp. 9-14, 2007.
[46] C. Eccleston, L. Hearn, and C. Williams. A. C. de, "Psychological therapies for the management of chronic neuropathic pain in adults," John Wiley \& Sons, Ltd 2015. 